\title{
Verifying Replicated Data Types with Typeclass Refinements in Liquid Haskell
}

YIYUN LIU, University of Maryland, College Park, USA

JAMES PARKER, University of Maryland, College Park, USA

PATRICK REDMOND, University of California, Santa Cruz, USA

LINDSEY KUPER, University of California, Santa Cruz, USA

MICHAEL HICKS, University of Maryland, College Park, USA

NIKI VAZOU, IMDEA Software Institute, Madrid, Spain

This paper presents an extension to Liquid Haskell that facilitates stating and semi-automatically proving properties of typeclasses. Liquid Haskell augments Haskell with refinement types-our work allows such types to be attached to typeclass method declarations, and ensures that instance implementations respect these types. The engineering of this extension is a modular interaction between GHC, the Glasgow Haskell Compiler, and Liquid Haskell's core proof infrastructure. The design sheds light on the interplay between modular proofs and typeclass resolution, which in Haskell is coherent by default (meaning that resolution always selects the same implementation for a particular instantiating type), but in other dependently typed languages is not.

We demonstrate the utility of our extension by using Liquid Haskell to modularly verify that 34 instances satisfy the laws of five standard typeclasses.

More substantially, we implement a framework for programming distributed applications based on replicated data types (RDTs). We define a typeclass whose Liquid Haskell type captures the mathematical properties RDTs should satisfy; prove in Liquid Haskell that these properties are sufficient to ensure that replicas' states converge despite out-of-order update delivery; implement (and prove correct) several instances of our RDT typeclass; and use them to build two realistic applications, a multi-user calendar event planner and a collaborative text editor.

CCS Concepts: • Software and its engineering $\rightarrow$ Formal software verification; Software verification; - Theory of computation $\rightarrow$ Distributed algorithms.

Additional Key Words and Phrases: replicated data types, CRDTs, typeclasses, refinement types, Liquid Haskell

\section{ACM Reference Format:}

Yiyun Liu, James Parker, Patrick Redmond, Lindsey Kuper, Michael Hicks, and Niki Vazou. 2020. Verifying Replicated Data Types with Typeclass Refinements in Liquid Haskell. Proc. ACM Program. Lang. 4, OOPSLA, Article 216 (November 2020), 30 pages. https://doi.org/10.1145/3428284

\section{INTRODUCTION}

Liquid Haskell [Rondon et al. 2008; Vazou et al. 2014] is an extension to Haskell that enables formally verifying logical properties of Haskell programs. Its basis for doing so is refinement types, which augment standard Haskell types with predicates that restrict the set of valid values [Rushby et al. 1998; Xi and Pfenning 1998; Constable and Smith 1987]; these predicates are checked automatically

Authors' addresses: Yiyun Liu, University of Maryland, College Park, USA; James Parker, University of Maryland, College Park, USA; Patrick Redmond, University of California, Santa Cruz, USA; Lindsey Kuper, University of California, Santa Cruz, USA; Michael Hicks, University of Maryland, College Park, USA; Niki Vazou, IMDEA Software Institute, Madrid, Spain.

This work is licensed under a Creative Commons Attribution 4.0 International License.

(C) 2020 Copyright held by the owner/author(s).

2475-1421/2020/11-ART216

https://doi.org/10.1145/3428284 
by an SMT solver. Liquid Haskell uses a mechanism called refinement reflection [Vazou et al. 2017] to lift the ability to state and check single refinements of individual functions to state and prove general properties of entire codebases [Vazou et al. 2018].

Liquid Haskell's features-being based on a widely used, industrial-strength programming language and having built-in support for proof automation-make it appealing as a platform for real-world, verified software development. Indeed, we were motivated to use it to build a platform for distributed computing applications employing data replication to increase reliability and availability. These applications are hard to get right, and we hoped Liquid Haskell's formal verification capabilities could help.

Unfortunately, there was a big stumbling block: Liquid Haskell cannot verify properties of typeclasses [Wadler and Blott 1989], which are used extensively throughout the Haskell ecosystem. Typeclasses in Haskell are similar to interfaces in Java or traits in Rust. A typeclass definition specifies a collection of method signatures that any type that is an instance of the typeclass must implement. For example, the Ord typeclass from Haskell's standard library declares that its instances a must have a method $(<=)$ of type a $\rightarrow$ a $\rightarrow$ bool; numbers, strings, booleans, and many other types are instances of Ord. The standard sort function can only sort lists of types that are Ord instances, since it needs a comparison function; this requirement is expressed as a constraint on sort's type, Ord a $\Rightarrow[a] \rightarrow[a]$.

Typeclass refinements for Liquid Haskell. The primary contribution of this paper is an extension to Liquid Haskell that supports stating and proving properties of typeclasses (Section 2). While it was previously possible in Liquid Haskell to prove properties of individual instances of a typeclass, it was not possible to give refinement types to a typeclass definition's methods. As such, Liquid Haskell code and proofs could not then modularly use those types when invoking methods from functions whose arguments (like sort) have a typeclass constraint. Given the ubiquity of typeclasses in Haskell code, the ability to do this is key to being able to verify interesting properties of real-world Haskell applications.

Implementing typeclass refinements in Liquid Haskell was not straightforward, which perhaps explains their long absence despite obvious benefits. Our implementation works by verifying properties not of Haskell source code, but rather of Core expressions, which are the intermediate representation produced by the Glasgow Haskell Compiler (GHC) [GHC 2020], the de facto Haskell standard. Doing so leverages functionality that GHC already provides (e.g., typechecking and elaboration) and allows Liquid Haskell to evolve semi-independently from GHC, since Core's definition is relatively stable. But there is a problem: typeclasses are not Core expressions-during elaboration, GHC translates them to dictionaries, which are basically records of functions. Code that defines a typeclass instance is translated to create a dictionary, and code that expresses a typeclass constraint is translated to use a dictionary; e.g., sort will be translated to be passed an Ord dictionary, from which it invokes the $(<=)$ method. To maintain the current separation between Liquid Haskell and GHC, our implementation (Section 3) transliterates typeclass methods' refinement types to checked invariants over dictionaries, so refinement types on typeclasses are verified when dictionaries are created, and those types can be used by client code. To do this modularly we had to expand the way Liquid Haskell interacts with GHC.

While Liquid Haskell is not the first proof system with typeclass support-Coq, Isabelle, Idris, $\mathrm{F}^{\star}$, Agda, and Lean have typeclasses or something like them-our approach represents an interesting point in the design space (see Section 5.3). A key element of a typeclass system is the resolution procedure, which selects an instance for a given type. For example, the code sort $[3,1,2]$ requires Haskell to resolve an instance of Ord at type Int so it can pass in the required Ord Int dictionary. Our support for typeclasses in Liquid Haskell reuses Haskell's typeclass resolution procedure. 
This procedure is coherent by default [Bottu et al. 2019], meaning that it always chooses the same typeclass instance for a given type (e.g., there cannot be two different implementations of Ord Int). The assumption of coherence can often be useful in proofs, especially when there are diamonds in the class inheritance graph. The above-listed languages do not ensure coherence, which can be awkward for the proof engineer; typeclass resolution may even fail to terminate. On the other hand, coherence is only the default in Haskell, not the rule: incoherent resolution (and overlapping instances) are sometimes useful, and enabled by pragma. As such, it would be unsound to axiomatize the assumption of coherence. Our implementation introduces a checked invariant during elaboration to express coherence, which Liquid Haskell proves automatically. This approach is novel and balances the extremes of accommodating full coherence or none. That said, reusing Haskell's resolution precludes different instances that refine the same base type,.g., an Ord instance for positive integers that is distinct from one for all integers. Fortunately, users can easily work around this limitation by defining instances on refined newtype wrappers, as is often done in Haskell anyway to avoid coherence problems (see Section 2.2).

Case study: Verifying standard typeclass laws. As a simple test of the utility of typeclass refinements, we carried out a small case study: We used Liquid Haskell to verify that instances of standard Haskell typeclasses satisfy the expected typeclass laws (Section 2.3). Significant prior work has focused on this application specifically, employing a variety of techniques, including random testing, term rewriting, contract verification, and conversion to Coq (see Section 5.2). Liquid Haskell typeclass refinements offer a natural, general-purpose approach. In particular, laws can be expressed as refinements to methods of a subclass of the target typeclass, and proofs of them are carried out in a subclass of each implementation of that target typeclass. This approach permits proofs of existing Haskell code without requiring that code be directly modified or annotated. We demonstrate this for several standard typeclasses, including Semigroup, Monoid, Functor, Applicative, and Monad, proving 34 instantiations satisfy their laws, in all (Section 2.3). Mostly, we find that the proofs are short (just a couple of lines), thanks to Liquid Haskell's SMT automation, and proof checking time is fast (typically a few seconds).

Case study: A platform for programming with verified replicated data types. Spurred by the success of this case study, we set out to build a platform for programming distributed applications based on replicated data types (RDTs) (Section 4). Data replication is ubiquitous in distributed systems to guard against machine failures and keep data physically close to clients who need it, but it introduces the problem of keeping replicas consistent with one another in the face of network partitions and unpredictable message latency. RDTs [Shapiro et al. 2011b,a; Roh et al. 2011] are data structures whose operations must satisfy certain mathematical properties that can be leveraged to ensure strong convergence [Shapiro et al. 2011b], meaning that replicas are guaranteed to have equivalent state given that they have received and applied the same unordered set of update operations.

Liquid Haskell typeclasses provide a natural, modular, and elegant way to implement and verify RDTs. We define a typeclass VRDT with a refinement type that captures the necessary properties, and we use Liquid Haskell to prove that those properties hold for a several primitive instances. We also define several larger VRDT instances by modularly combining both the code and proofs of smaller ones. We state and prove, in Liquid Haskell, the strong convergence property that VRDT instances enjoy. Pleasantly, our approach generalizes and relaxes the typical assumption of causal message delivery. Our VRDT instances are sufficiently expressive that with them we were able to build a shared calendar event planner and a collaborative text editor. Each application is implemented using a few hundred lines of Haskell code (Section 4.7).

Although there exists previous work on mechanized verification of many RDTs (Section 5.4), ours are, we believe, the first immediately executable, mechanically verified implementations of 
multiset, two-phase map, and causal tree [Grishchenko 2010] RDTs. Because Liquid Haskell is an extension of standard Haskell, our applications are real, running Haskell applications, but now using mechanically verified RDT implementations.

Contributions. In summary, this paper makes the following contributions:

- We present an extension to Liquid Haskell that supports stating and proving refinements of typeclass methods' types. The engineering of this extension is an interesting interaction between GHC and Liquid Haskell's core proof infrastructure, and our design sheds light on the interplay between coherent typeclass resolution and modular proofs (Sections 2 and 3).

- We use our extension to Liquid Haskell to modularly verify that 34 standard instances satisfy the laws of five widely-used Haskell typeclasses, the Semigroup, Monoid, Functor, Applicative, and Monad typeclasses (Section 2.3).

- We further use our extension to Liquid Haskell to implement a platform for distributed applications based on verified replicated data types. We define a typeclass whose Liquid Haskell type captures the mathematical properties that must be true of RDTs, and we prove in Liquid Haskell that strong convergence holds if these properties are satisfied (Section 4).

- We implement (and prove correct) several instances of our refined typeclass, including the first immediately executable, mechanically verified implementations of multiset, two-phase map, and causal tree [Grishchenko 2010] RDTs (Section 4.2).

Using these verified RDT instances,${ }^{1}$ we implement two realistic applications: a shared calendar event planner and a collaborative text editor (Section 4.7).

As of this writing, we have nearly completed merging our extension into the main Liquid Haskell implementation, at which point it will be freely available.

\section{TYPECLASSES IN LIQUID HASKELL}

This section begins with background on Liquid Haskell [Rondon et al. 2008; Vazou et al. 2014], which extends Haskell with refinement types (Section 2.1). Then it presents our extension to Liquid Haskell, which permits annotating a typeclass definition's methods with refinement types, thus allowing a typeclass's clients to assume those richer types, while obligating a typeclass's instances to implement them (Section 2.2). As a demonstration of the effectiveness of this approach, we verify that 34 instances of five standard typeclasses satisfy the expected laws (Section 2.3).

\subsection{Refinement Types and Liquid Haskell}

A refinement type augments a base type $T$ with a predicate $\phi$ that restricts the set of valid values [Rushby et al. 1998; Xi and Pfenning 1998; Constable and Smith 1987]. In Liquid Haskell, a refinement type has the form $\{x: T \mid \phi\}$-the base type $T$ is refined according to predicate $\phi$, which may refer to values of the base type via the variable $x$ (if it appears free in $\phi$ ). For example, a refinement type for positive integers would be $\{\mathrm{x}$ : int $\mid \mathrm{x}>0\}$, i.e., $T=$ int and $\phi=\mathrm{x}>0$.

In Figure 1, the function head uses this kind of refinement on the type of its input list xs, stating the precondition that the list's length be positive. This refinement thus prevents calling head with an empty list, thus precluding the exception that could otherwise result.

Also in the figure we see code for Haskell's standard list append operator, (++), which uses a refinement to state a postcondition. The (standard) code states that appending an empty list [] with a list ys yields ys (line 2), while appending a non-empty list (with a head element $\mathrm{x}$ and a tail xs) with a list ys is the result of cons'ing $x$ to the front of $x$ s appended to ys (line 3). The refinement type states that the output list's length is equal to the sum of the lengths of the input

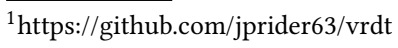




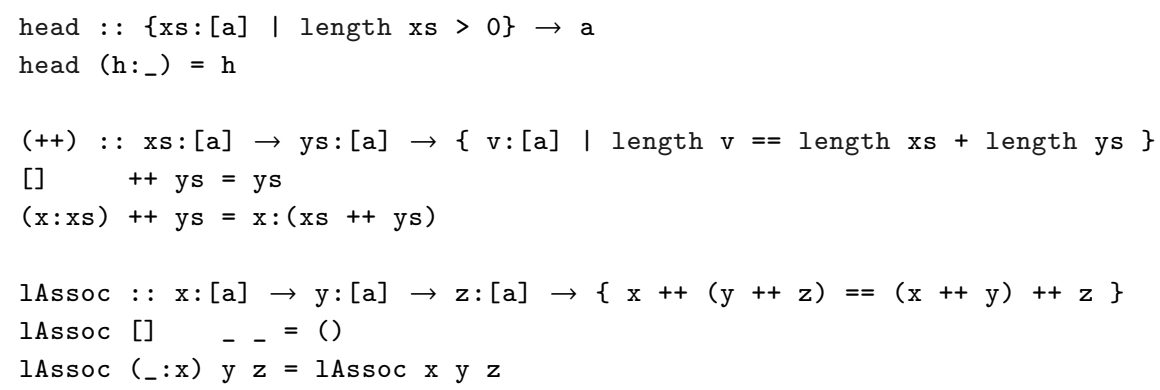

Fig. 1. Haskell's list head and append (++) functions augmented with refinement types to capture pre- and post-conditions; and 1 Assoc, a statement and proof that append is associative.

lists. The refinement type predicate is able to refer to the function's inputs via names xs and ys, which annotate the parameters' types. Liquid Haskell proves that postconditions such the one on $(++)$ hold by generating appropriate verification conditions from the code and delegating to an SMT solver (in particular, Z3 [De Moura and Bjørner 2008]); we say more on this below.

In the refinement types of head and (++), length refers to the Haskell length function on lists. Such references to normal language terms are lifted into the refinement logic through a process called refinement reflection [Vazou et al. 2017]. Refinement reflection uses the definitions of Haskell's functions to generate singleton refinement types that precisely describe the result of the function. To ensure soundness of type checking, only provably terminating functions can be reflected.

Refinement reflection makes it possible to write and mechanically verify proofs of independent, general properties, e.g., involving many functions and not just a single one. These are called extrinsic properties, as they are written externally to any particular function's definition, as opposed to intrinsic properties like the ones on head and (++). For example, 1Assoc in Figure 1 is an extrinsic property (and proof) that append is associative. The property is the type, which states that for all lists $\mathrm{x}, \mathrm{y}, \mathrm{z}$ we have that $\mathrm{x}++(\mathrm{y}++\mathrm{z})$ equals $(\mathrm{x}++\mathrm{y})++\mathrm{z}$. Note that the postcondition of lAssoc is equivalent to $\{\mathrm{v}$ : unit $\mid \mathrm{x}++(\mathrm{y}++\mathrm{z})==(\mathrm{x}++\mathrm{y})++\mathrm{z}\}-$ the $\mathrm{v}$ : unit part is dropped since there is no need to name the result, which is not mentioned in the predicate.

Proofs of extrinsic properties are themselves Liquid Haskell definitions whose type is the desired property. The proof in our example is the body of 1 Assoc, which expresses that the property holds by induction-the base case is for [] and the recursive case is for $\left({ }_{-}: \mathrm{x}\right)$ y $\mathrm{z}$. In the base case, there is nothing specific the programmer has to write other than (), the "return type" of the property For the recursive case, the inductive argument occurs by referring to the property on the strictly smaller input $\mathrm{x}$ y $\mathrm{z}$ (rather than $\left({ }_{-}: \mathrm{x}\right)$ y $\mathrm{z}$ ). This proof follows a standard formula [Vazou et al 2018] in which the handwritten part shown here provides the structure, and the proof details are filled in using a combination of Proof by Logical Evaluation (PLE) [Vazou et al. 2017; Leino and Pit-Claudel 2016] to automate function unfolding, and SMT solving, which automates reasoning over specific theories (e.g., equality and linear arithmetic). Both strategies preserve decidable type checking. Such automation helps make it possible to write substantial proofs in Liquid Haskell, as previously demonstrated with a proof of noninterference for the LWeb system [Parker et al. 2019]. Proofs can also be done by hand, as needed/desired [Vazou et al. 2018].

Liquid Haskell's implementation is simplified by making use of GHC, the Glasgow Haskell Compiler [GHC 2020], to partially evaluate programs. Liquid Haskell first parses refinement types, which are written in the comments of normal Haskell code. Then it passes the Haskell code to GHC, and gets back the code as Core, which is GHC's intermediate language. Liquid Haskell lifts the Core 


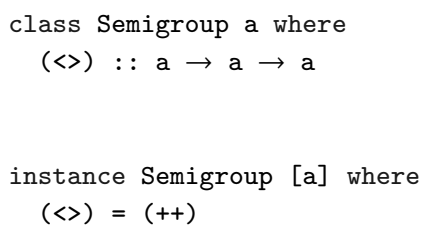

(a) Standard Semigroup typeclass and the list instance of it

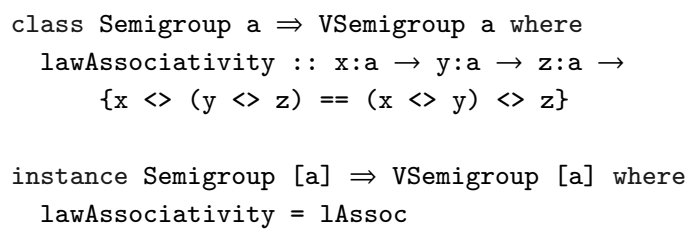

(b) VSemigroup extends Semigroup with an associativity law, which its list instance satisfies via IAssoc

Fig. 2. Typeclasses with Refinement Types

output into the refinement logic using refinement reflection. Finally, it converts the refinement types and corresponding Core output into SMT-LIB2 queries [Barrett et al. 2010] which can automatically be verified by Z3. If any queries are invalid, Liquid Haskell reports an error message.

\subsection{Refinement Types for Typeclasses}

We have extended Liquid Haskell to allow typeclass methods to be annotated with refinement types. Doing so allows a developer to state properties that a typeclass's methods should always satisfy. Clients of that typeclass can thus assume those properties in their own proofs. Of course, implementors of the typeclass's instances must prove the properties hold for their instance.

Laws as Refinement Types. We illustrate the utility of our extension by showing how standard typeclass laws can be encoded as refinement types. Laws are properties that clients of a typeclass generally assume, and that implementors of a typeclass are supposed to ensure. Of course, without something like our extension, there is no guarantee that they do so.

Figure 2(a) shows the Semigroup typeclass, which defines a type a that is equipped with a single operator $\langle>$. One particular implementation of this typeclass for lists ([a]) is also shown, where <> corresponds to the List append operator. A key law of semigroups is that the <> operator is associative. Clients of Semigroup may assume this law holds of any instance they are given; they may break if it does not. Fortunately, as we proved in the previous subsection, List append is associative, so the List instance of Semigroup satisfies the law. How can we show this?

We extend the syntax of typeclasses to allow for refinement types on method declarations. Below is a version of Semigroup extended to capture the associativity typeclass law as a refinement type.

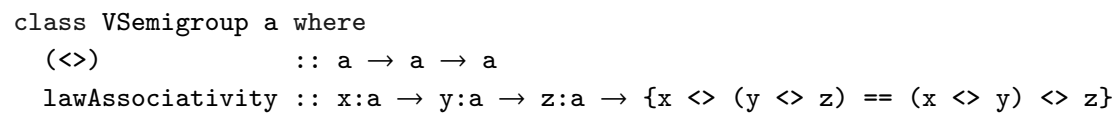

VSemigroup matches the definition of Semigroup from Figure 2(a) but adds typeclass method lawAssociativity, which (extrinsically) defines the associativity property. All vSemigroup instances are now required to define lawAssociativity and provide an explicit associativity proof. The lower portion of Figure 2(b) implements the list instance of vSemigroup by extending Semigroup list instance and providing the associativity proof lAssoc from Section 2.1.

Using the Laws, Modularly. By allowing refinement types on typeclass definitions, we extend the modularity benefits of typeclasses from code to proofs. In particular, clients of a refined typeclass can take advantage of its stated refinement types when conducting their own proofs. For example, below we express and prove an extrinsic property that extends associativity to four elements.

assoc2 : : VSemigroup $\mathrm{a} \Rightarrow \mathrm{x}: \mathrm{a} \rightarrow \mathrm{y}: \mathrm{a} \rightarrow \mathrm{z}: \mathrm{a} \rightarrow \mathrm{w}: \mathrm{a}$ 


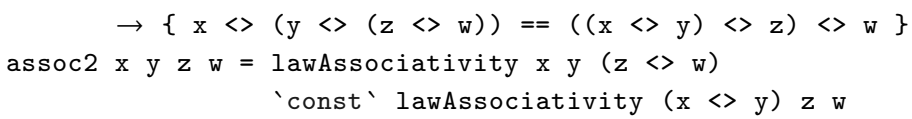

The proof is a consequence of lawAssociativity, which is applied twice, combined with Haskell's constant function. The proof is carried out once, independent of any VSemigroup instance, but the property holds for all of them.

The code of our VRDT case study (Section 4) is set up similarly. We define a VRDT typeclass with operations on data that enjoy particular properties. Relying on these properties, we can prove strong convergence of all VRDTs; this property essentially states that two replicas that start in the same state will end up in the same state if they apply the same operations, in any order.

Refinements in Subclasses. For improved modularity, our extension allows typeclass method refinements to refer to superclass methods. For example, another way to write VSemigroup is shown at the top of Figure 2(b), which literally extends Semigroup with the added method. Defining properties in subclasses is particularly useful when not wanting to modify typeclasses in other packages (including those in normal, not Liquid, Haskell). It can also be useful when not wanting to necessarily require implementations to prove all possible properties; different subsets of properties of interest can be defined in different subclasses.

Haskell typeclasses can have multiple superclasses, which allows defining a typeclass containing properties of data structures that implement multiple typeclasses. For example, consider the Monoid typeclass, which extends Semigroup to also include the mempty identity element. Since a particular data structure (like a list) can implement both typeclasses, we could define the verified typeclass VMonoid that extends VSemigroup and Monoid with two laws.

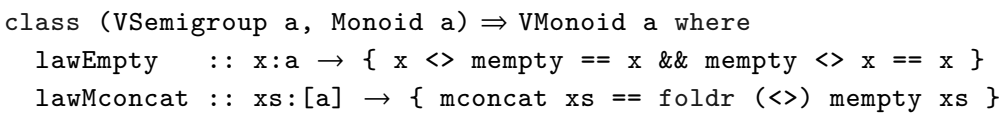

That mempty is an identity for $<>$ is encoded in the lawEmpty method; it refers to $(<>)$, which is defined in the vSemigroup parent typeclass. The law lawMconcat guarantees that mconcat, defined by Monoid, is equivalent to folding over a non-empty list with $(<>)$.

We can also define verified components from other verified components, where proofs of the former's properties can depend on properties that hold of the latter. For example, in our VRDT case study, we define a VRDT TwoPMap in terms of any other VRDT; here is the beginning of the instance definition:

instance (Ord k, VRDT v) $\Rightarrow$ VRDT (TwoPMap k v) where ...

The proofs of TwoPMap $\mathrm{k}$ v's properties make use of the properties that hold for Ord $\mathrm{k}$ and VRDT v.

Coherent Typeclass Resolution and Refined Instances. There is an interesting twist in our vMonoid example. As mentioned, Monoid extends Semigroup; as such, proofs of properties in VMonoid may wish to assume that the VSemigroup instance resolved for VMonoid has the same parent superclass as that of the resolved Monoid instance. Indeed, this assumption is critical for the given properties: we require that the <> operator in both Monoid and vSemigroup to be literally the same function.

Our implementation reuses Haskell's existing typeclass resolution procedure, which is useful from an engineering perspective, and has two consequences. First, and importantly for the above example, Haskell typeclass resolution is coherent by default, but not as a rule. That resolution is coherent means that there is only one possible typeclass instance for a particular base type, e.g., Semigroup [a] will always resolve to the same instance definition. Coherence is handy for addressing the above "diamond problem" [Stroustrup 1989]. That said, programmers may override coherence via pragma. While doing so is rare, the possibility means that axiomatizing an assumption 
of coherence would be unsound. Our implementation therefore introduces a checked invariant on an elaborated dictionary when coherence could be consequential, i.e., because the class in question has multiple parent superclasses that should have a common ancestor. Liquid Haskell then automatically proves this invariant after resolution has taken place. We give more details about how this works in Section 3.3.

The second consequence of reusing Haskell's resolution procedure is that we cannot directly support instances on refined types, only base types. For example, we cannot have distinct semigroup instances for positive and negative numbers, i.e., instance vSemigroup $\{\mathrm{v}$ : Int $\mid 0<\mathrm{v}\}$ and instance VSemigroup $\{\mathrm{v}$ : Int $\mathrm{V}<0\}$.

Fortunately, there is an easy workaround: users can define instances on refined newtype wrappers; newtype is already commonly used in Haskell to offer an alternative instance implementation while maintaining coherence. So, for example, a user could define:

newtype Pos $=$ Pos $\{$ getPos $::\{\mathrm{v}:$ Int $\mid \mathrm{v}>0\}\}$

newtype Neg $=$ Neg $\{$ getNeg : : $\{\mathrm{v}:$ Int $\mid \mathrm{v}<0\}\}$

Now we can define the VSemigroup instances without any additional refinements, as follows:

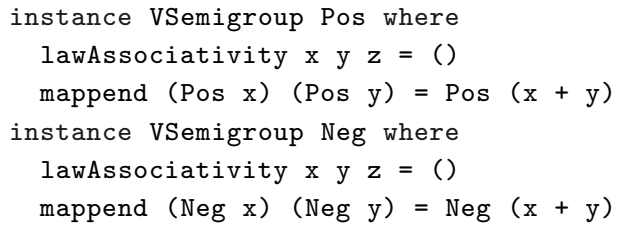

\subsection{Verifying Laws of Standard Typeclass Instances}

Before getting into the details of how we implemented typeclass refinements (in the next section) we present a case study demonstrating that the pattern we have shown for stating and verifying the laws of standard typeclass instances works well.

In our case study, we considered five standard typeclasses: Semigroup, Monoid, Functor, Monad, and Applicative. Then we defined subclasses (VSemigroup, VMonoid, etc.) that contain the parent's expected typeclass laws. We have shown the definitions of vMonoid and vSemigroup already; Functor, Monad, and Applicative are shown in Figure 3 with their refined subclasses. We defined and verified instances of the above typeclasses for the All, Any, Dual, Endo, Identity, List, Maybe, Peano, Either, Const, State, Reader, and Succs datatypes. Because datatypes are instances of multiple subclasses, we performed 34 instance-verifications in total.

This effort was quite manageable. Table 1 tabulates the results, indicating the instance type in the first column, and the typeclasses it implements in the second. For each implementation we tabulate the lines of proof required to verify the stated laws. We also report the average (and standard deviation) of the time (in seconds) it took Liquid Haskell to verify each module. ${ }^{2}$

For many of the proofs, Liquid Haskell is able to automatically verify the typeclass properties using PLE (Proof by Logical Evaluation) [Vazou et al. 2017; Leino and Pit-Claudel 2016]. As such, most of the proofs are a couple of lines of code. In general, PLE reduces manual effort but increases verification time, but for most modules the proofs are checked within just a few seconds. There are some exceptions-the List, Reader, and Succs Applicative instances are more involved as they require the user to manually specify certain lemmas.

\footnotetext{
${ }^{2}$ Experiments were run with five trials. All experiments in this paper were run on a machine with an Intel Xeon CPU with 64GB of RAM, running Ubuntu 16.04 with Z3 version 4.8.8.
} 


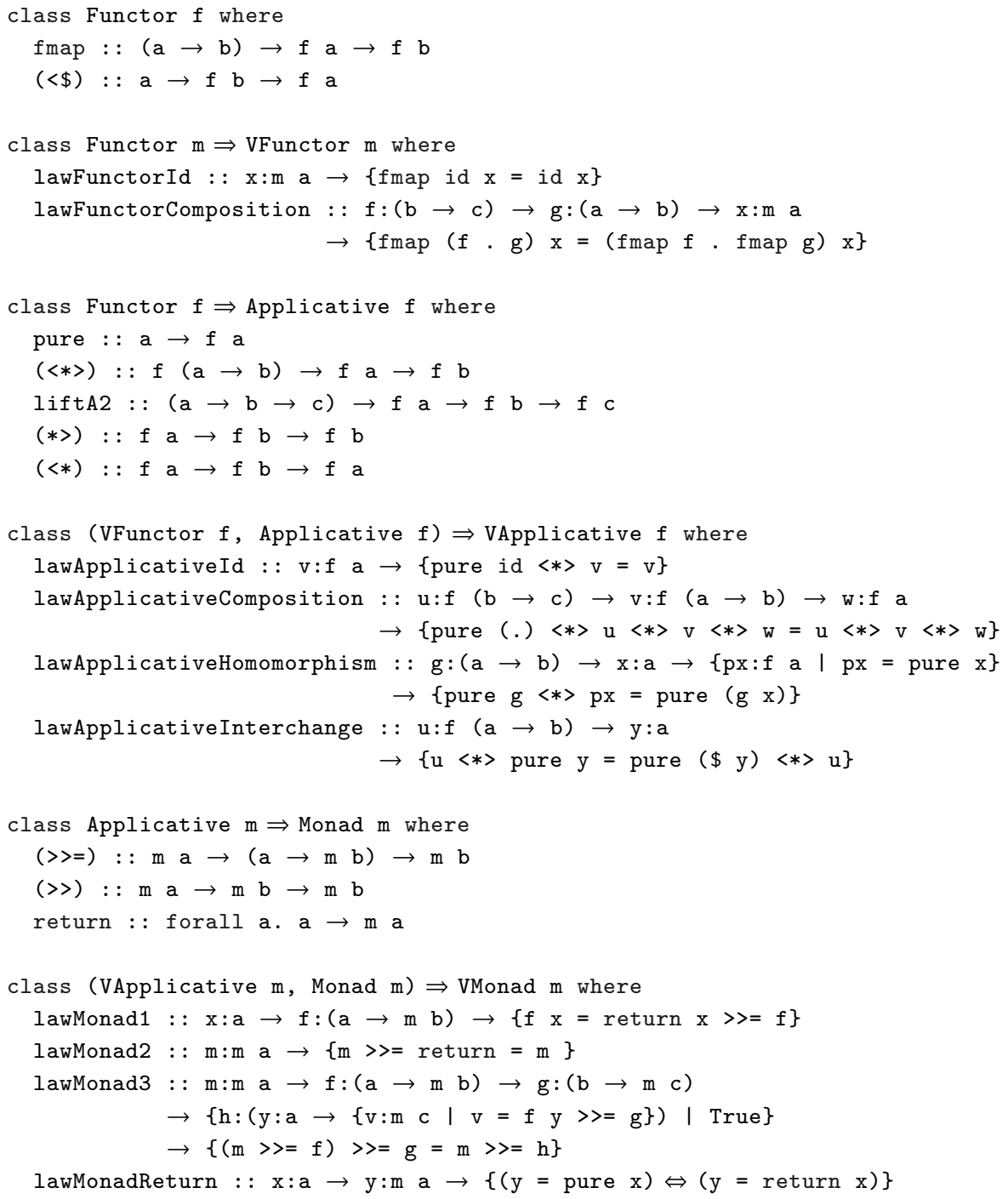

Fig. 3. Typeclass definitions for Functor, Applicative, and Monad and their associated laws.

In sum, this case study shows that typeclass refinements constitute a natural and modular approach to stating typeclass laws and proving that they are satisfied by their instances. Section 4 presents further evidence, in the form of our VRDT case study, of the utility of typeclass refinements.

\section{IMPLEMENTING TYPECLASS REFINEMENTS}

Now we turn to the question of how we extended Liquid Haskell to implement typeclass refinements.

Liquid Haskell statically verifies Haskell programs by analyzing Core expressions. Core is a small, explicitly-typed variant of System F generated during compilation by GHC, the Glasgow Haskell Compiler. Liquid Haskell can thus ignore many of Haskell's myriad source-level constructs, and focus on a smaller language. This implementation approach is also useful for managing Liquid 
Table 1. Total lines of proofs for each typeclass instance and the average verification time in seconds. Each reported time covers the laws on its row and those on the following rows up to the next reported time.

\begin{tabular}{llrr}
\hline Type & Typeclass & $\begin{array}{r}\text { \# Lines } \\
\text { Proof }\end{array}$ & $\begin{array}{r}\text { Verif. Time } \\
\text { (Std. dev.) }\end{array}$ \\
\hline All & Semigroup & 2 & $1.555(0.133)$ \\
& Monoid & 2 & \\
Any & Semigroup & 2 & $1.129(0.112)$ \\
& Monoid & 2 & \\
Enal & Semigroup & 2 & $1.522(0.254)$ \\
& Monoid & 2 & \\
Identity & Semigroup & 2 & $1.326(0.191)$ \\
& Monoid & 2 & \\
& Semigroup & 2 & $1.534(0.186)$ \\
& Monoid & 2 & \\
& Functor & 2 & $2.727(0.274)$ \\
& Applicative & 4 & \\
List & Monad & 4 & \\
& Semigroup & 3 & $1.436(0.148)$ \\
& Monoid & 3 & \\
& Functor & 4 & $8.117(0.380)$ \\
& Applicative & 25 & \\
& Monad & 10 & \\
\hline
\end{tabular}

\begin{tabular}{llrr}
\hline Type & Typeclass & $\begin{array}{r}\text { \# Lines } \\
\text { Proof }\end{array}$ & $\begin{array}{r}\text { Verif. Time } \\
\text { (Std. dev.) }\end{array}$ \\
\hline Maybe & Semigroup & 3 & $4.360(0.500)$ \\
& Monoid & 3 & \\
& Functor & 3 & $3.961(0.428)$ \\
& Applicative & 8 & \\
& Monad & 6 & \\
Peano & Semigroup & 3 & $1.236(0.157)$ \\
& Monoid & 3 & \\
Either & Functor & 3 & $4.672(0.538)$ \\
& Applicative & 8 & \\
& Monad & 6 & \\
Const & Functor & 2 & $0.530(0.077)$ \\
State & Functor & 12 & $1.182(0.118)$ \\
Reader & Functor & 11 & $2.997(0.347)$ \\
& Applicative & 21 & \\
Succs & Functor & 2 & $6.390(0.649)$ \\
& Applicative & 18 & \\
\hline & & & \\
\hline
\end{tabular}

Haskell as an independent codebase. Even as Haskell is actively modified with new or improved features, Liquid Haskell needs no modification because those features are translated to Core.

The challenge with implementing typeclass refinements is that GHC removes typeclasses entirely during the translation to Core; each typeclass is replaced with a dictionary of its various operations. Thus, our extension to Liquid Haskell needs a way to connect the refinements the programmer writes on typeclass methods with the translated Core that comes back from GHC, and it needs to do so in a way that is robust to (at least some) future changes in GHC's elaboration. This section explains how we do this by delegating as much work as possible to GHC. We also explain how we model the fact that typeclass elaboration is coherent by default, to simplify user proofs.

\subsection{GHC Typeclass Elaboration}

Haskell compilers, including GHC, translate typeclass definitions and instances to datatypes known as dictionaries [Sulzmann et al. 2007]. As an example, the Semigroup typeclass definition from Figure 2(a) is translated to a dictionary as the following datatype, Semigroup (simplified for clarity).

data Semigroup a $=$ CSemigroup $\{(<>):: a \rightarrow a \rightarrow$ a $\}$

The datatype Semigroup a has a single constructor CSemigroup and one field for the <> method. In general, one field is defined for each typeclass method.

Typeclass instances are translated into dictionary values. For example, the list Semigroup instance from Figure 2(a) generates a Semigroup [a] dictionary, which GHC names \$fSemigroup [].

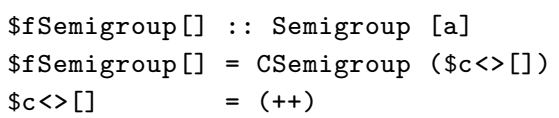

The dictionary's field is the list append method $(++)$, which is assigned to the generated variable $\$ c<>[]$. (Both the dictionary and field variables are prefixed with $\$$ to indicate they are internal variable names, and posfixed with [] to indicate the list instance.) 
Elaboration. The translated dictionaries are inserted after each method call via a process known as elaboration. For example, the Haskell code $\mathrm{x}\langle>\mathrm{y}$ that appends two list variables $\mathrm{x}, \mathrm{y}:$ : [a] is elaborated to $(<>)$ \$fSemigroup [] $\mathrm{x}$ y, where now $(<>)$ is the record selector of the Semigroup data type. Functions that explicitly mention the Semigroup a constraint, as in $f$ below, are elaborated to take an explicit dictionary argument; $f$ elaborates to $\mathrm{fElab}$, on the right.

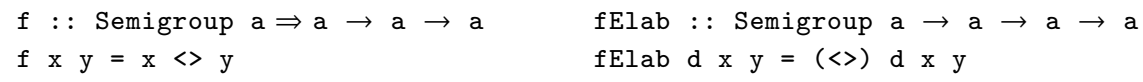

Subclass Encoding and Coherence. In Core, subclass dictionaries store references to parent dictionaries as fields. For example, the dictionary of the VMonoid typeclass from Section 2.2 has four fields, two for the class methods and two for the superclass dictionaries:

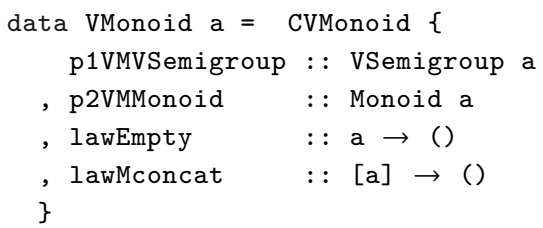

Interestingly, Semigroup is a superclass of both Monoid and VSemigroup, which leads to the "diamond problem." When the user writes $\mathrm{x}<>\mathrm{y}$, it is unclear if GHC's elaboration will access $(<>)$ via the Monoid or via the vSemigroup field. That is, GHC can elaborate the coherence code below to either coherenceElab1 or coherenceElab2 .

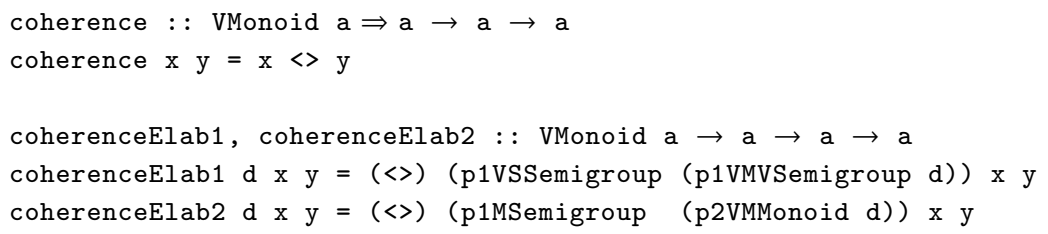

Here, p1VSSemigroup and p1MSemigroup access the semigroup dictionary from the VSemigroup and Monoid, respectively. Such nondeterminism of elaboration could lead to problems, as the runtime semantics of coherence could change with GHC's elaboration decision. Fortunately, by default GHC's elaboration is coherent [Bottu et al. 2019], meaning that the dictionary for each typeclass instance at a given type is unique; as such, we know that the Semigroup dictionary is the same irrespective of how it is accessed, i.e., ( 1VSSemigroup . p1VMVSemigroup $)=($ p1MSemigroup - p2VMMonoid ). Such an equality may be needed in a proof, so our implementation reflects it (in a safe manner) in the proof state, as discussed in Section 3.3.

\subsection{Interaction with GHC}

Now we explain how we modified Liquid Haskell's interaction with GHC so that we can verify typeclass refinements.

Refinements are ported to Dictionaries. The core intuition of typeclass verification is that refinements on typeclasses should be turned into refinements on the respective GHC-translated dictionaries. For example, the dictionary for the vSemigroup refined typeclass of Figure 2(b) should be refined to carry the associativity proof obligation (as a normal refinement):

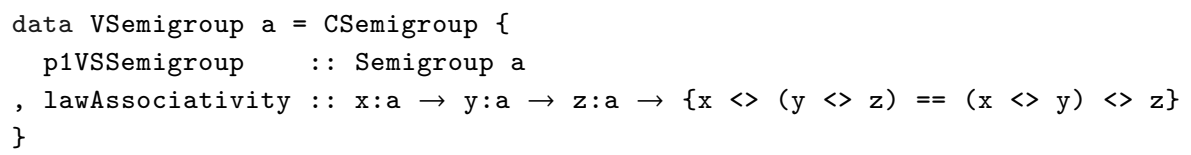




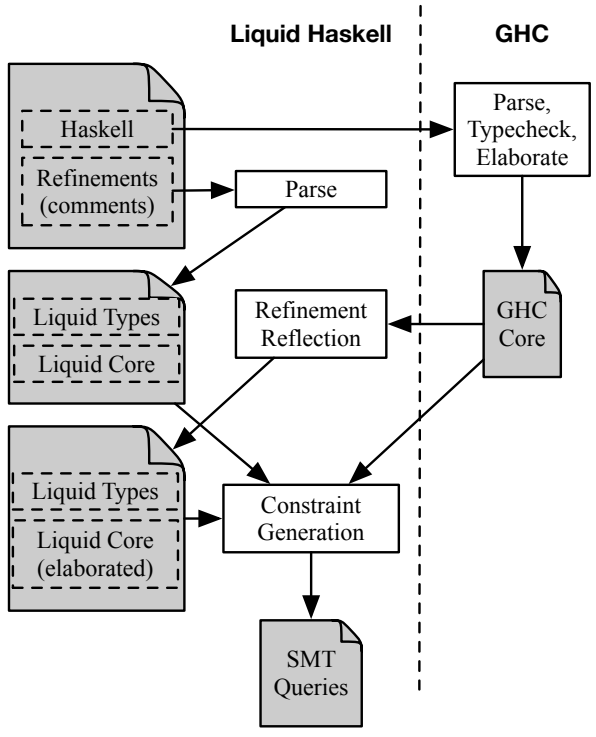

(a) Liquid Haskell's original architecture.

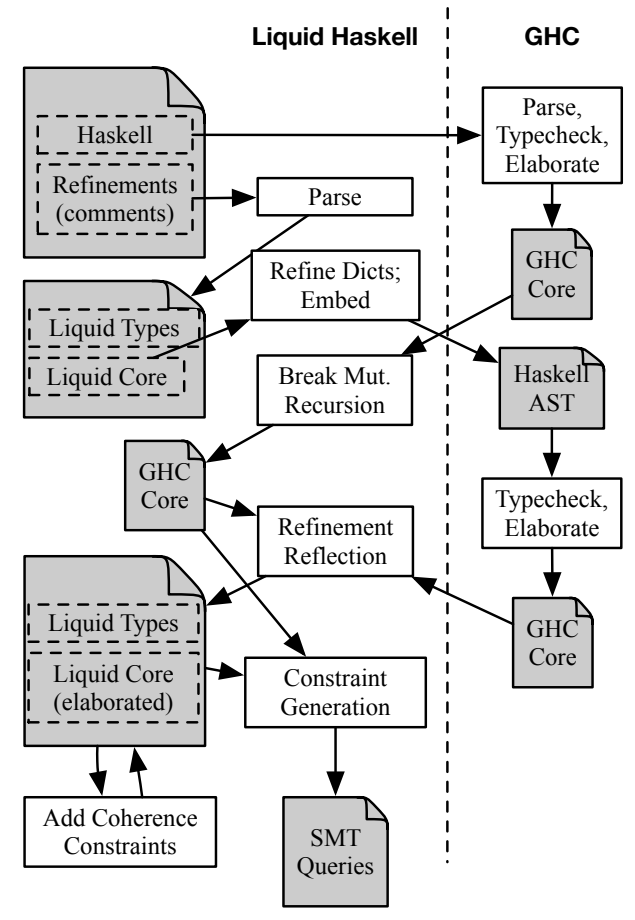

(b) Liquid Haskell's architecture with typeclass support.

Fig. 4. Changes to Liquid Haskell's architecture.

(Here, the first field of the dictionary is a link to the parent typeclass.) But of course we cannot literally do this because lawAssociativity is not well-formed Core. We make it so by expanding Liquid Haskell's interaction with GHC, using it to parse, typecheck, and elaborate refinements.

Liquid Haskell's Architecture. Figure 4 summarizes Liquid Haskell's architecture and interaction with GHC API before and after support for refined typeclasses.

The first step is similar in both architectures: send Haskell source to GHC, which comes back as Core, and parse refinement types appearing in comments. Before typeclass support (Figure 4a), the Core expressions were used by Liquid Haskell to strengthen the exact types for reflected functions and to generate the verification constraints that were finally checked by an SMT. After typeclass support, the returned Core may include dictionary definitions, elaborated implementations of those dictionaries, and elaborated clients that use them, as explained in Section 3.1. These dictionaries need to be connected to the refinement types retrieved from typeclass methods.

To connect typeclass method refinements to elaborated dictionaries, Liquid Haskell converts the parsed-out refinements into Haskell abstract syntax trees that make explicit reference to the relevant typeclasses. This occurs in the Refine Dicts; Embed step of the architecture diagram. As an example, for the VSemigroup method's refinement of lawAssociativity (Figure 2), the following Haskell source-code AST expression is constructed:

$(\backslash \mathrm{x}$ y $\mathrm{z}() \rightarrow \mathrm{x} \ll(\mathrm{y}\langle\mathrm{z})=(\mathrm{x}<>\mathrm{y})<>\mathrm{z}):$ : VSemigroup $\mathrm{a} \Rightarrow$

$\mathrm{a} \rightarrow \mathrm{a} \rightarrow \mathrm{a} \rightarrow($ ) $\rightarrow$ Bool 
GHC typechecks and elaborates the expression in the context of the vSemigroup definition and applies the appropriate dictionary arguments to typeclass methods. It returns the following:

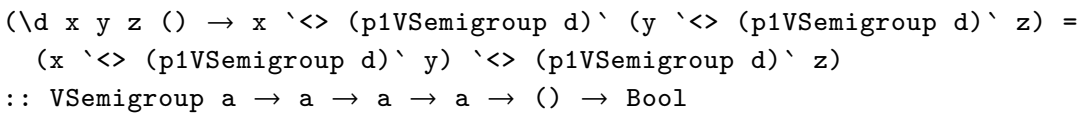

Now the dictionary $d$ is explicit in the elaborated Core expression. Liquid Haskell converts this into a refinement expression using refinement reflection, and then combines it with the Core code returned from GHC in the first step; for the example, the constructor and selectors for VSemigroup in Figure 2 are the following:

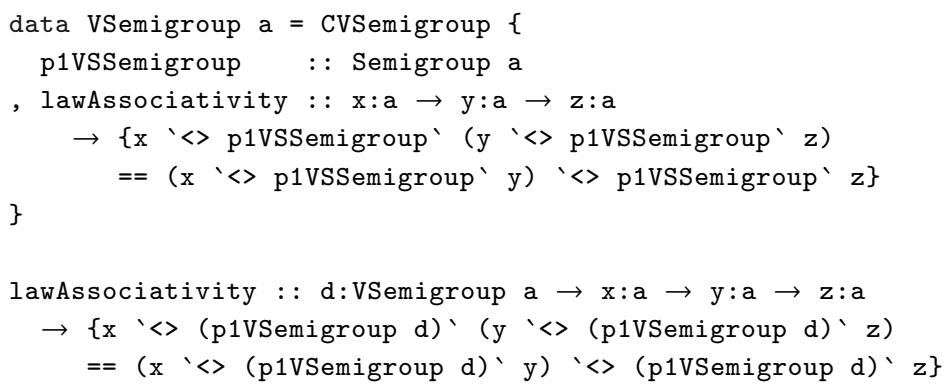

First, notice that lawAssociativity basically matches the elaborated Core expression returned by GHC, but it has been converted to match Liquid Haskell's refinement type syntax. Second, notice that the VSemigroup data constructor uses the returned Core, but has applied one additional step of transformation so that it can refer to the superclass' (i.e., Semigroup) operator. Now, VSemigroup instances must satisfy the required properties since the dictionary datatype has been refined.

Typeclass methods do not only appear in the refinements of typeclasses. Functions with typeclass constraints may also contain typeclass methods in their refinements. We also elaborate the refinement expressions of these functions so that the appropriate dictionary arguments to typeclass methods are applied.

This whole process corresponds to the refine dicts; embed, typecheck, elaborate, and refinement reflection steps in the diagram. The breaking mutual recursion step inlines selector calls in the derived GHC dictionaries to break superficial mutual recursion since Liquid Haskell requires explicit proof of termination. The adding coherence constraints step is detailed in Section 3.3.

Our implementation of all of this amounts to about 2000 lines of code, and is part of a fork Liquid Haskell's codebase which is up to date with the main trunk as of August, 2020. Around 400 lines of code is used to define functions that communicate with the GHC API. The top-level driver function that orchestrates GHC's Typecheck; Elaborate and Liquid Haskell's Refine Dicts; Embed step takes another 700 lines of code. This also includes the embedding functions from Liquid Haskell predicates to the Haskell AST. The rest of the code roughly corresponds to the Refinement Reflection step, where elaborated dictionaries are being converted into ordinary refined data types which Liquid already knows how to process and verify.

\subsection{Reasoning About Coherence}

In Section 3.1 we mentioned that GHC's elaboration is, by default, coherent. Various proofs on typeclass methods rely on coherence of elaboration (i.e., only hold when instances are globally unique). Here, we give an example of such a proof and detail how coherence is automatically encoded and checked using refinement types. 
lawMconcat for the VMonoid Dual Instance Requires Coherence. Given any binary operation <>, we can always define a dual operation $\langle+\rangle$ :

$\mathrm{x}\langle+>\mathrm{y}=\mathrm{y}\langle>\mathrm{x}$

Therefore, if we have a Semigroup instance for some type a, we can also define a Dual instance of a Semigroup. Indeed, we can define Semigroups of Duals of any type a once and for all:

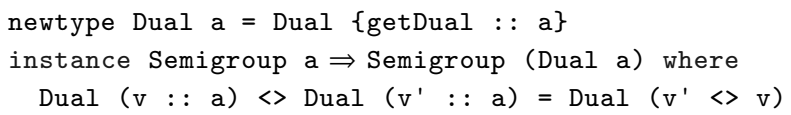

Now, whenever we define a Semigroup instance for some type, GHC will automatically create its corresponding Dual instance. We do the same for Monoid, VSemigroup, and VMonoid.

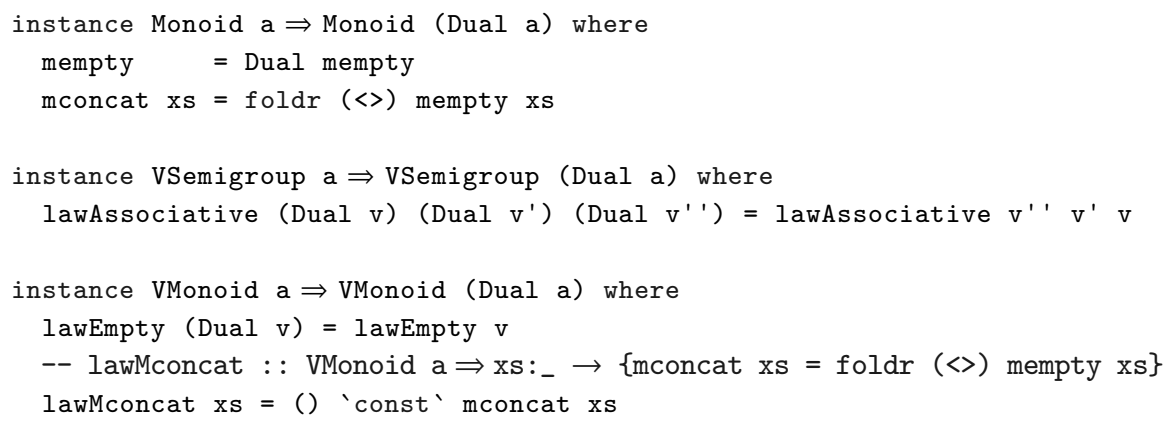

The proof of lawMconcat proceeds by a simple unfolding of the mconcat definition, which is expressed as a call to that function (the full proof must be then cast to unit via Haskell's const ()).

This proof requires coherence to hold. To see why, consider the proofs for the elaborated definitions. The elaborated equality mconcat $x s=f o l d r \quad(<>)$ mempty $x s$ is as follows, for the dictionary d : : VMonoid a.

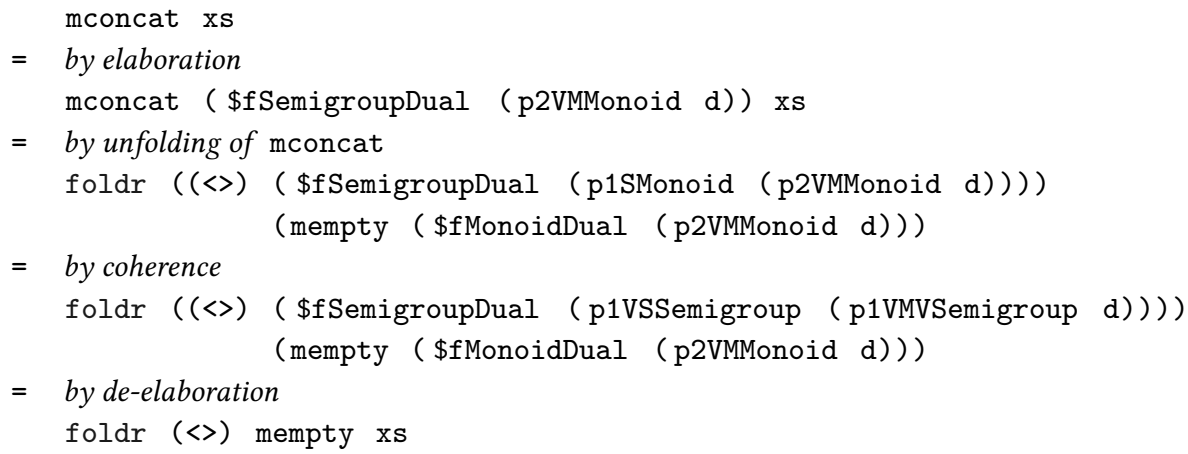

The proof employs a coherence step to equate the two different ways that the semigroup operator $<>$ is accessed. Concretely, the above equational proof only holds when p1VSSemigroup ( 1VMVSemigroup d) equals p1SMonoid (p2VMMonoid d), for all d : : VMonoid a. This equality cannot be asserted by the programmer, as dictionaries do not appear in the source.

Coherence as Dictionary Refinements. We represent the expected effect of coherent resolution as a refinement type on the datatype dictionary definitions for typeclasses. In particular, the equality of ancestor typeclasses is expressed as a refinement type on the fields of parent typeclasses. For example, the Core representation of VMonoid is as follows. 


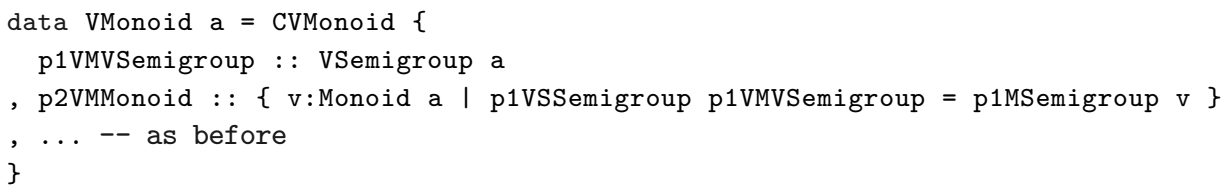

The added refinement on the second field states that the Semigroup from the VSemigroup parent (i.e., p1VSSemigroup p1VMVSemigroup) must be equal to the Semigroup from the Monoid parent (i.e., p1MSemigroup v). (This invariant is similar in purpose to to an ML-module sharing constraint [MacQueen 1984].) As a result, coherence becomes a checked invariant for each VMonoid-Liquid Haskell assumes the property for any VMonoid but checks it for instance declarations. This approach is sound even when GHC's elaboration is potentially incoherent due to use of the INCOHERENT language pragma. Clients of dictionaries will still assume the invariant, but constructed dictionaries (i.e., typeclass instances) will induce an error from Liquid Haskell if the choice made by incoherent resolution breaks the invariant. We note that inheritance diamonds are rare in Haskell, so such checked invariants will also be rare.

Being able to take advantage of Haskell's (mostly) coherent typeclass resolution is the silver lining of our limitation that refined types cannot be distinct typeclass instances, as discussed at the of end of Section 2.2. If we allowed different instances of a class TC for both $\{\mathrm{v}$ : int $\mid \mathrm{v}>0\}$ and int, say, then we could not use Haskell's proved-coherent mechanism, and would have to develop our own and/or allow one to be customized as part of proof search. There is no guarantee that the result would be coherent. In other dependently typed systems with typeclasses, e.g., Coq, the user has to explicitly encode and prove coherence requirements, case by case (see Section 5).

\subsection{Incompatibility with SMT interpreted Operators}

Predating our extension's support for typeclass refinements, Liquid Haskell provided specialized support for the Eq, Ord, and Num typeclasses by hard-coding their expected behavior. For example, in the case of Eq, Liquid Haskell interprets uses of the == operator from the Eq typeclass as SMT equality, which amounts to structural equality in Haskell. This specialized support allows the programmer to reason about equality, ordering, and numbers in Liquid Haskell programs, and leverages the SMT solver's automation. Unfortunately, it is neither sound nor complete. In the case of Eq, soundness fails because a user-defined equivalence relation does not necessarily imply the stronger structural equality. In the case of Ord, completeness fails when a concrete Ord instance is collapsed into an abstract partial order, losing the extra information from the instance definition.

We might imagine this legacy specialized handling can now be dropped in favor of typeclass refinements. Unfortunately, such an approach would break existing proofs, even for properties that were not affected by the potential unsoundness. This is because there would no longer be a connection between the user-defined instances of the three classes and their corresponding SMT theories, thus hampering automated reasoning. The SMT solver is unable to recognize that the post-elaboration expression (+) $\$$ fNumFoo (the + method of the Foo instance of Num) can be reasoned about abstractly using the solver's internal knowledge of the axioms of numbers; the method (+) is merely a ternary operation (when including the dictionary argument), like any other. Of course, the programmer can start from the class laws and derive lemmas manually, but that is not what is happening in current proofs. And indeed, we want to let the SMT solver automatically derive those properties using its decidable theories.

As such, at present we continue to ignore the instances from the aforementioned classes, e.g., by discarding the dictionary argument and interpreting the instances specially. In the future we plan to explore a hybrid approach that leverages SMT theories when possible (e.g., when we can prove soundness and retain precision) and falls back to general (dictionary-based) refinements otherwise. 


\section{CASE STUDY: VERIFIED REPLICATED DATA TYPES}

This section presents our platform for programming distributed applications based on replicated data types (RDTs) [Shapiro et al. 2011b,a; Roh et al. 2011]. We define a Haskell typeclass VRDT to represent RDTs and use type refinements to state the mathematical properties that RDTs satisfy. We then prove in Liquid Haskell that VRDT instances (which must satisfy these properties) enjoy strong convergence.

We have implemented several primitive instances of the VRDT typeclass, as well as a mechanism for building compound VRDTs based on smaller components, where the necessary properties of the former automatically follow from the latter. With this infrastructure, as well as libraries for message delivery and user interaction we developed, we constructed two applications, a shared event planner and a collaborative text editor.

\subsection{Background: Replicated Data Types}

An RDT is a data structure designed for use in a distributed system that stores multiple replicas of the same data. The mathematical properties of an RDT ensure the strong eventual consistency (SEC) of replicas [Shapiro et al. 2011b], the most important aspect of which is strong convergence (SC). SC states that RDT replicas that have received and applied the same set of updates will always have the same state, regardless of the order in which those updates were received and applied.

Shapiro et al. [2011b] describe two styles of RDT specifications: state-based, in which replicas apply updates locally and periodically broadcast their local state to other replicas, which then merge the received state with their own, and operation-based, in which every state-updating operation is broadcast and applied at each replica. Shapiro et al. [2011b] prove that state-based and operation-based RDTs are equivalent in the sense that each can be implemented in terms of the other (although practical implementation considerations may motivate the choice of one or the other in a particular application). In this work, we focus our attention on the operation-based style, which is especially suitable for implementing ordered sequence RDTs that are useful for applications such as collaborative text editing [Roh et al. 2011; Ahmed-Nacer et al. 2011; Attiya et al. 2016; Oster et al. 2006; Preguica et al. 2009; Weiss et al. 2009; Kleppmann and Beresford 2017]

The key to proving convergence of (operation-based) RDTs is to require that, under appropriate circumstances, an RDT's operations commute. A replica that receives an operation from a client can update its own state and broadcast that operation to the other replicas. Since the operations commute, they can be applied in any order and produce the same final state, as required by SC.

\subsection{Verifying Strong Convergence of RDTs using Typeclass Refinements}

We define an RDT as the Liquid Haskell typeclass VRDT, shown in Figure 5(a). Each instance of VRDT has an associated Op type that specifies the operations that can update the RDT's state. The apply method takes an RDT state $t$, runs the given operation Op $t$ on it, and returns the updated state. Read operations on an RDT do not affect convergence, so they are not included in $\mathrm{Op}$; rather, each VRDT instance defines its own read methods.

The required mathematical properties of a VRDT are specified extrinsically as methods lawCommut and lawCompatCommut. The type of lawCommut specifies the property that operations that are compatible with each other and with the current state must commute. The type of lawCompatCommut expresses that the operation-compatibility predicate compat must also be commutative. The implementor of a VRDT instance must provide definitions for the compat and compatS predicates that lawCommut and lawCompatCommut use. The notion of operation compatibility will depend on the RDT in question, and compat and compatS should encode any necessary assumptions. For example, in our TwoPMap key-value map RDT implementation given in Figure 6, we express the assumption 


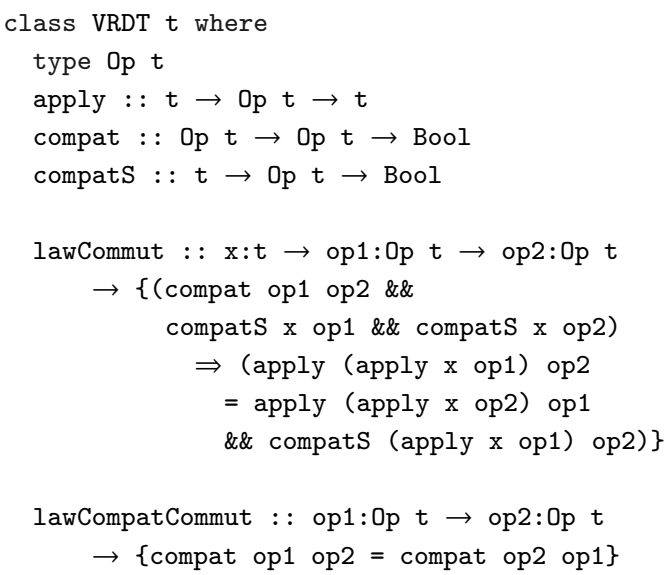

(a) The VRDT typeclass for verified RDTs

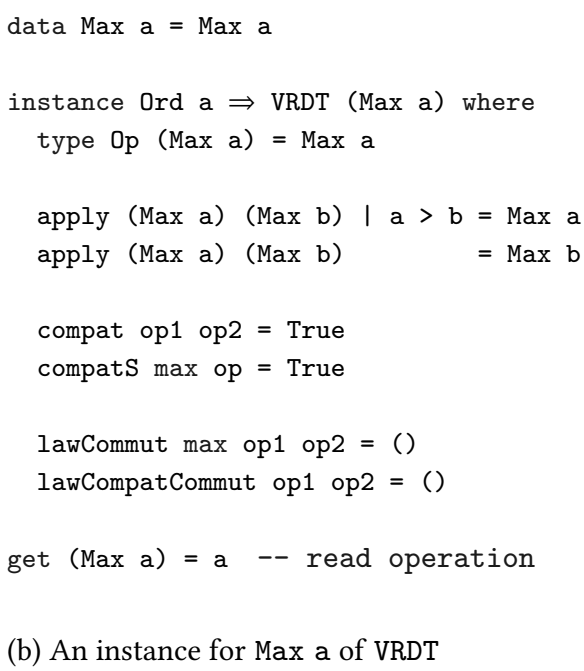

(b) An instance for Max a of VRDT

Fig. 5. Definition of the VRDT typeclass and its Max instance

of unique keys in compat and compats by deeming two insertion operations incompatible if they specify the same key, and deeming an insertion incompatible with a state that already contains the specified key. In the Max RDT implementation described next, compat and compatS both return True, meaning that no assumptions are needed in order to prove that Max operations commute.

Example VRDT. Figure 5(b) gives an example VRDT instance, Max. It contains a polymorphic value with a defined ordering (specified with an Ord instance) and tracks the maximum value of that type. Its corresponding operation's type $0 \mathrm{p}$ is itself. The apply function updates Max's state by taking the greatest value of the two arguments. The get method is Max's read operation. Because Max is such a simple RDT, all pairs of operations are compatible, so compat and compatS always return True. Proofs of lawCommut and lawCompatCommut for Max are automated by Liquid Haskell.

Strong Convergence. All VRDT instances enjoy strong convergence, the key safety property required by strong eventual consistency [Shapiro et al. 2011b], expressed extrinsically as follows:

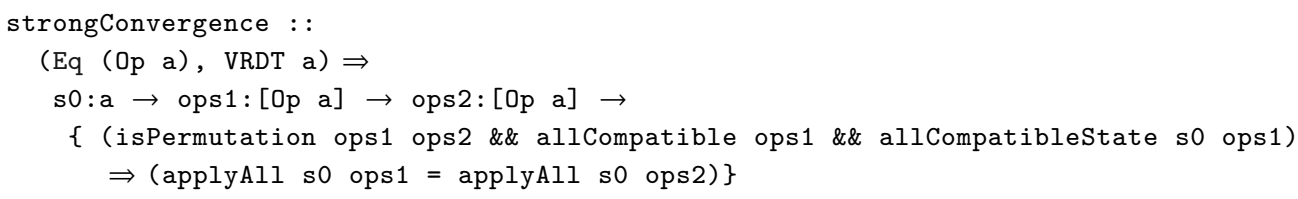

The property states that if two lists of operations are permutations of one another, then applying either one to the same input VRDT state will produce the same output state, assuming the list contains mutually compatible operations, and that all of these operations are compatible with the initial state. The Liquid Haskell proof of this property is by induction over the operation lists and makes use of the lawCommut and lawCompatCommut laws of VRDT. Importantly, the proof is independent of any particular VRDT instance, and thus applies to all of them.

Unlike existing work [Shapiro et al. 2011b; Gomes et al. 2017], we do not rely on any assumptions about so-called causal delivery-the given laws are sufficient for proving strong convergence. We discuss further in Section 4.5. 


\subsection{Implemented VRDTs}

In addition to Max, we have implemented and mechanically verified four more primitive VRDTs:

- Min $\mathrm{v}$ is the dual of Max $\mathrm{v}$, and tracks the smallest value seen.

- Sum v is an implementation of Shapiro et al. [2011a]'s Counter RDT. Ops are numbers, and the RDT's state is their sum.

- LWW $t \mathrm{v}$ is an implementation of Shapiro et al.'s Last Writer Wins Register RDT. When a replica writes to a register, it attaches a (polymorphic) timestamp to the value. A receiving replica only updates its value if the timestamp is greater than the current timestamp. LWW assumes that all timestamps are unique.

- MultiSet v maintains a collection of values, like a Set, but each member has an associated count; a non-positive count indicates logical non-membership. Ops include value insertion and removal, each with an associated count.

Causal Trees. More substantially, we have also implemented Grishchenko's causal trees [2010], which maintain an ordered sequence of values. In a CausalTree, each value is assumed to have a unique identifier, and each value knows the identifier of the previous value. The relationship to the previous value creates a tree data structure that can be traversed (in preorder) to recover a converging ordering. Causal trees, like other RDTs representing ordered sequences (e.g., Roh et al [2011]; Oster et al. [2006]; Preguica et al. [2009]; Weiss et al. [2009]), are useful for implementing collaborative text editing applications, but their behavior is considered especially challenging to specify and verify [Attiya et al. 2016; Gomes et al. 2017]. We discuss our proof in Section 4.4.

A Compound RDT: Two-phase Map. We can conveniently define new VRDTs by reusing existing VDRTs. In doing so, proofs of a compound RDT's required properties can be carried out (in part) by using the properties of the RDTs they build on. As an example compound VRDT, Figure 6 defines a two-phase map. TwoPMap implements a map from keys to values, where values are themselves VRDTs. TwoPMap is named after the $2 P$-Set of [Shapiro et al. 2011a] because similarly, a key may be inserted or removed, but cannot be reinserted once it has been removed.

A TwoPMap's operations are given by the datatype TwoPMap0p. Operation TwoPMapInsert $\mathrm{k} v$ inserts a $\mathrm{k} \rightarrow \mathrm{v}$ mapping; operation TwoPMapDelete $\mathrm{k}$ deletes a key; and TwoPMapApply $\mathrm{k}(\mathrm{Op} \mathrm{v}$ ) applies a VRDT operation Op on k's value v.

The restriction of TwoPMap which gives its name, that a key can only be inserted once and after it is deleted it can never be re-added, is expressed in the definitions of compat and compatS. These methods of the TwoPMap VRDT instance reiterate the requirement that the operations of the value $V R D T$ are compatible, connecting the proofs into one. A few additional cases are omitted from the compatibility predicates for brevity.

The state of a TwoPMap has three components: the key-value map itself ( $t$ woPMap), a tombstone set of deleted keys ( twoPMapTombstone ) [Shapiro et al. 2011a], and a buffer for pending operations (twoPMapPending). The apply code for TwoPMapApply stores operations on the value of a key $\mathrm{k}$ in the pending buffer if $\mathrm{k}$ does not yet exist in the map. The apply code for TwoPMapInsert checks the pending buffer for the key to be inserted and applies any operations on the given value before inserting the key/value pair. The apply code for TwoPMapDelete clears $\mathrm{k}$ from the map and operations buffer, and adds it to the tombstone set; future attempted insertions of $\mathrm{k}$ will be ignored due to its presence there.

Automatically Deriving Compound VRDTs. Generally speaking, we might like to collect together several VRDTs to create an aggregate whose operations delegate to the operations of the components. For example, the shared event planner we discuss in Section 4.7 represents a calendar event as a record with a title, description, time, and guest RSVP tally. To implement such a record as a VRDT, 


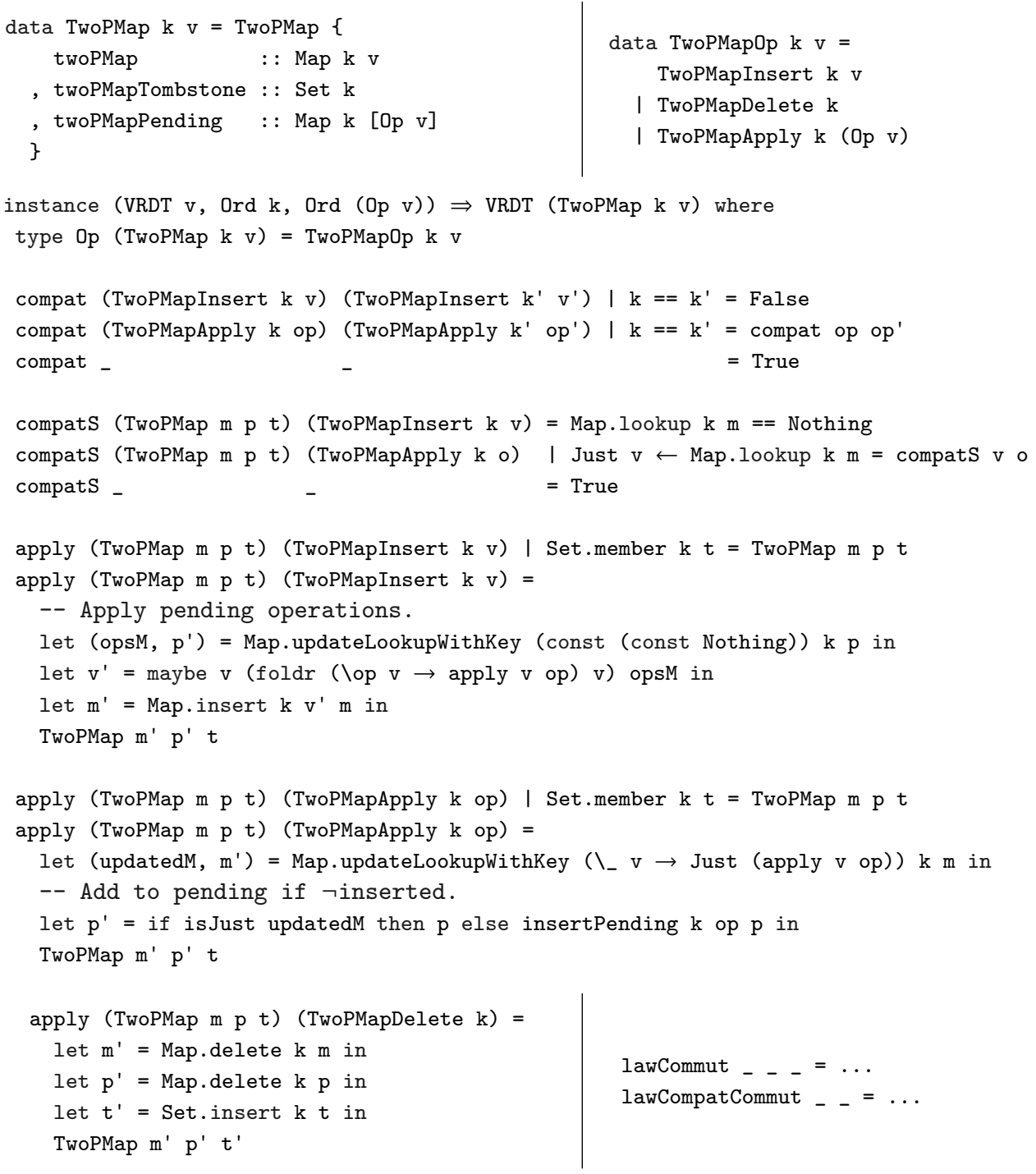

Fig. 6. Implementation of TwoPMap.

we can represent the first three fields as LWW registers and the last as a MultiSet, as shown in the upper left of Figure 7. However, just collecting separate VRDTs together is not enough to show that the result is a VRDT: we need to define a corresponding Op data type and a VRDT instance for Event. Fortunately, since the fields of Event are VRDT instances, it is possible to derive the Event operation and VRDT instance automatically. We use Template Haskell [Sheard and Peyton Jones 2002] to, at compile time, generate operations and VRDT instances for data types that are composed of other VRDTs. The rest of Figure 7 shows the automatically generated code for Event. EventOp is its operation data type and each instance method, including the lawCommut and lawCompatCommut laws, is defined recursively for each field. Liquid Haskell automatically verifies that the generated code satisfies the VRDT properties. 


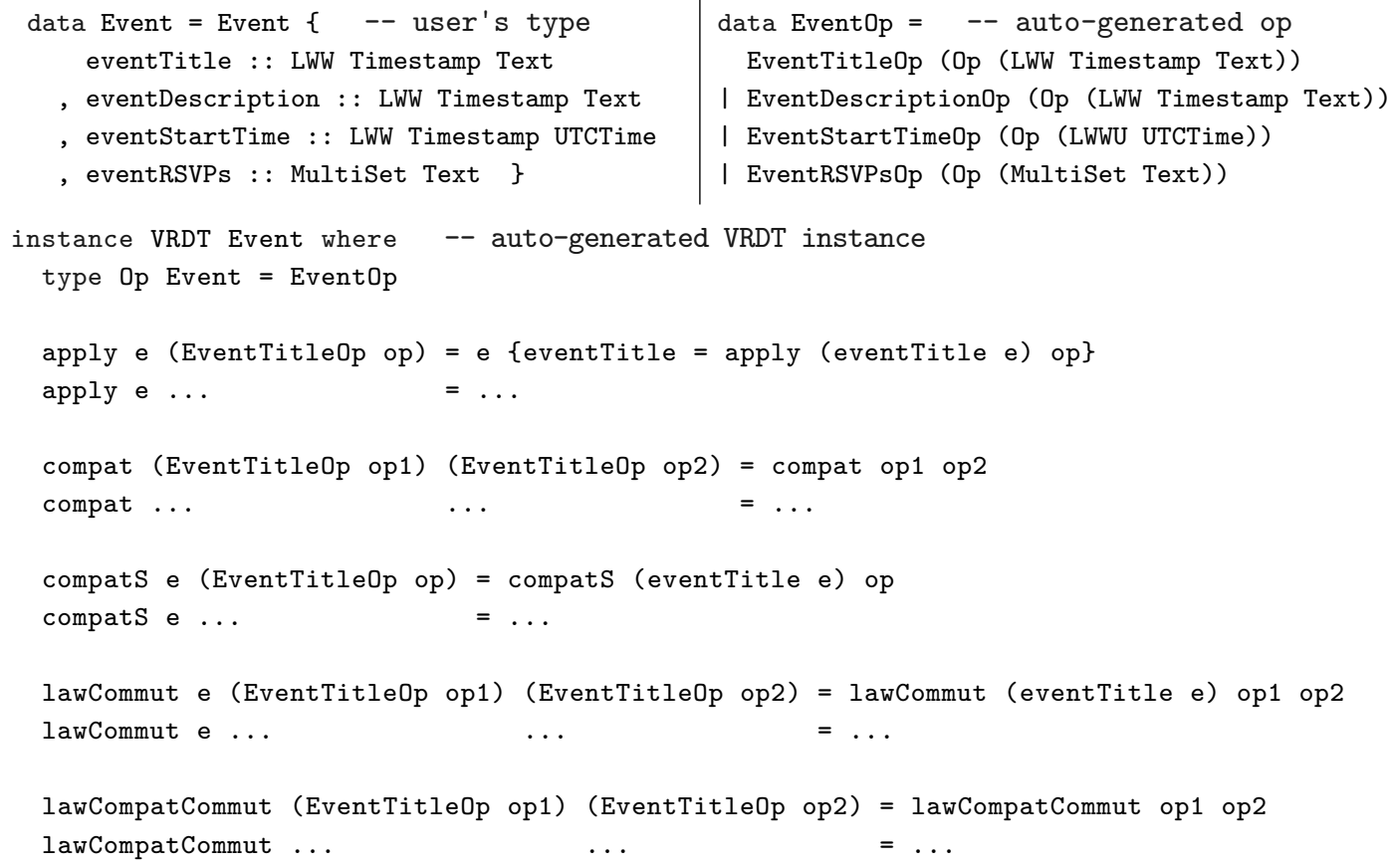

Fig. 7. Data type Event for a calendar event (upper left), and the automatically generated operation data type (EventOp) and VRDT instance for Event.

\subsection{Verification Effort}

A VRDT instance enjoys strong convergence as long as it satisfies lawCommut and lawCompatCommut, which must be proved for its particular implementation. Table 3 summarizes the lines of proof and verification time for the VRDT instances we built. The development totals 5536 lines of code. These also include duplicate definitions of Haskell functions to be amenable to verification. For example, we redefined Data. Map to prove it satisfies the invariant that its keys are sorted, while common list functions were redefined to be reflected, as required by extrinsic proofs. As expected, Liquid Haskell's PLE and SMT automation over intrinsic properties (e.g., sortedness invariant on Data . Map) aided proof generation. That said, there are still some issues to iron out. For example, we had difficulties proving properties of code that makes use of typeclasses that have SMT-interpreted theories in Liquid Haskell, e.g., set theory used by the verified Data . Map.

The proof of TwoPMap also ran very slowly; because of the large search space (nine case splits between the three operations), the verification took more than three hours. The long verification time can be attributed to PLE's expansion and the discharging of verification conditions by the SMT solver. The bloated verification conditions consume a significant amount of memory space as well; when verifying the insert/apply case of TwoPMap, Liquid Haskell exhausted the $16 \mathrm{GiB}$ physical memory and consumed no less than $1 \mathrm{GiB}$ of the swap space. Interestingly, the proof for TwoPMap relies on the strongConvergence property that holds for all VRDT instances. This property is needed since the values in a TwoPMap are inhabitable by any VRDT instance. We used strongConvergence to show that operations in the pending buffer can be applied in any order.

The commutativity proof for CausalTree was tricky. Each operation depends on another operation: When a node is inserted, its dependent nodes in the pending queue are recursively applied. 


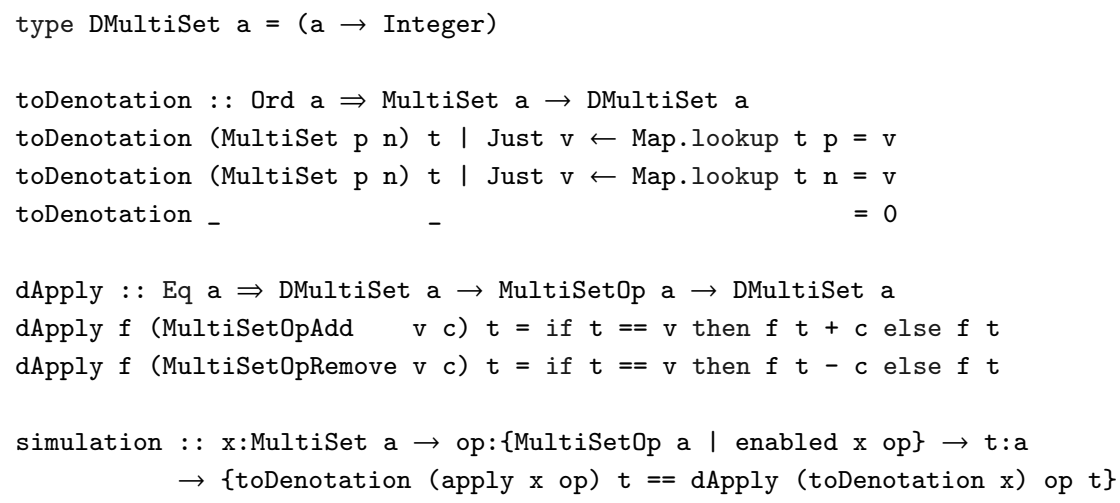

Fig. 8. Denotational semantics of Multiset.

The resulting tree of dependencies induces significant case-based reasoning. For example, suppose we want to show that op1 and op 2 commute, where the parent of op 1 is in the CausalTree and the parent of op2 is pending. If op2 is applied first, then clearly op2 will be inserted into the pending queue. If op1 is applied first, we cannot be sure that op2 will still be pending since descendants of the pending operations of op1 could be the parent of op2. Handling each case where one operation may be the ancestor of another operation was difficult.

Just because a data type satisfies the required VRDT typeclass laws does not mean its implementation is correct. An advantage of Liquid Haskell is that it is also possible to specify and verify higher level properties about RDTs. To demonstrate this, we prove that the behavior of our Multiset VRDT simulates the mathematical (denotational) semantics of multisets (Figure 8). Multiset maintains a positive map $\mathrm{p}$ and a negative map $\mathrm{n}$; the former contains members with positive counts while the latter contains members with non-positive counts. The Ops are MultiSetOpAdd and MultiSetOpRemove ; they shift a value between maps as its count crosses 0 . We define the denotation of a MultiSet to be a function from an element of the Multiset to the number of copies of that element. This is represented by the type alias DMultiSet. The toDenotation function is a straightforward mapping from a MultiSet to a DMultiSet that looks up the element in the positive and negative Map's of the MultiSet. dApply defines how to run a MultiSet operation on a DMultiSet by adding the number of new copies to the existing count. The DMultiSet denotation serves as a simple specification of how we expect Multiset to operate. We prove that Multiset and DMultiSet have the same behavior: The simulation theorem states that for all of a MultiSet's enabled operations, looking up an element when you apply the operation on the MultiSet and then convert it to its denotation returns the same result as when you first convert it to a DMultiSet and run the operation on the denotation.

In summary, the verification effort was strenuous, which was expected as the first real-world case study of refined typeclasses. Nevertheless, this case study increases our confidence that Liquid Haskell's automation reduces proof effort and, since most of the implementation limitations we faced are already addressed, that refined typeclasses in Liquid Haskell can actually be used to verify sophisticated properties of real-world applications.

\subsection{Discussion: Causal Delivery of RDT Operations}

In typical developments of RDTs, only concurrent operations are required to commute, where concurrent operations are those not ordered by the happens-before partial order [Lamport 1978], 
Table 3. Total lines of proofs for each typeclass instance and the average verification time in seconds. Verification times for lawCommut and lawCompatCommut are combined.

\begin{tabular}{llrr}
\hline VRDT & Property & $\begin{array}{r}\text { \# Lines } \\
\text { Proof }\end{array}$ & $\begin{array}{r}\text { Verif. Time } \\
\text { (Std. dev.) }\end{array}$ \\
\hline- & strongConvergence & 320 & $777.079(344.937)$ \\
\hline \multirow{2}{*}{ Max } & lawCommut & 1 & $2.565(1.046)$ \\
& lawCompatCommut & 1 & \\
\multirow{3}{*}{ Sum } & lawCommut & 1 & $2.8194(1.106)$ \\
& lawCompatCommut & 1 & \\
& lawCommut & 1 & $2.185(0.676)$ \\
& lawCompatCommut & 1 & \\
\hline
\end{tabular}

\begin{tabular}{llrr}
\hline VRDT & Property & $\begin{array}{r}\text { \#Lines } \\
\text { Proof }\end{array}$ & $\begin{array}{r}\text { Verif. Time } \\
\text { (Std. dev.) }\end{array}$ \\
\hline LWW & lawCommut & 1 & $4.125(1.742)$ \\
Multiset & lawCompatCommut & 1 & \\
& lawCommut & 315 & $136.639(30.309)$ \\
& lawCompatCommut & 1 & \\
TwoPMap & simulation & 72 & $121.849(45.478)$ \\
& lawCommut & 1253 & $11487.275(197.175)$ \\
CausalTree & lawCompatCommut & 3 & \\
& lawCommut & 2402 & $11242.311(111.787)$ \\
& lawCompatCommut & 1 & \\
\hline
\end{tabular}

also known as the causal order. Such an assumption may place additional requirements on the underlying communication mechanism. For example, Shapiro et al. [2011b] assume that updates will be delivered to operation-based RDT replicas in causal order, e.g., by employing vector clocks [Fidge 1988; Mattern 1989] and buffering received operations until all operations that causally precede them have been applied [Birman et al. 1991]. Shapiro et al. further assume that any preconditions that must be satisfied to enable an operation's execution (e.g., that a key must be present in a map if its value is to be updated) are already ensured by causal delivery.

As mentioned earlier, our VRDT convergence proof does not rely on any assumption of causal delivery, but in turn requires a proof that all operations commute, not just concurrent ones, as stated by lawCommut and lawCompatCommut. We took this approach because requiring causally ordered delivery of updates is neither necessary nor sufficient for strong convergence [Nagar and Jagannathan 2019], and removing the assumption frees the communication mechanism from implementing causal delivery. In turn, this shrinks the size of our trusted computing base compared to other RDT verification work, e.g., Gomes et al. [2017].

On the other hand, not assuming causal delivery may add complication to the RDT implementations, and increase the proof burden, in some cases. For simpler VRDT instances such as Max, this is not so: all operations commute anyway, so taking away the assumption of causal delivery posed no problem for verification. For other instances, such as TwoPMap, proving lawCommut and lawCompatCommut required us to put more care into how the apply operation and the compat and compatS predicates were implemented. Indeed, the pending buffer used by apply in our TwoPMap implementation is reminiscent of the buffering mechanism that an implementation of causal delivery would use. The additional burden of these challenges can be reduced by reusing abstractions like pending buffers and vector clocks (and their corresponding proofs) across multiple RDTs.

\subsection{Performance}

One advantage of a Liquid Haskell-based verification of RDTs is that the object of verification is executable Haskell code. As such, there are no hidden costs in the translation from the verified artifact to the executable one. To demonstrate this, we benchmark the median runtime of applying a single operation on our VRDT implementations versus the number of operations that have been applied to the data structure. For each VRDT, we compare against a corresponding baseline, nonreplicated Haskell data structure, and we randomly generate operations to apply. The baseline for TwoPMap and MultiSet is the canonical Data. Map (Figure 9a). For these data types, 60\% of operations are insertions, $20 \%$ of operations are updates, and $20 \%$ of operations are deletions. CausalTree's baseline is Data. List where $70 \%$ of operations are insertions and $30 \%$ of operations are deletions (Figure 9b). We ran 11 trials, and all measurements were performed on the same 

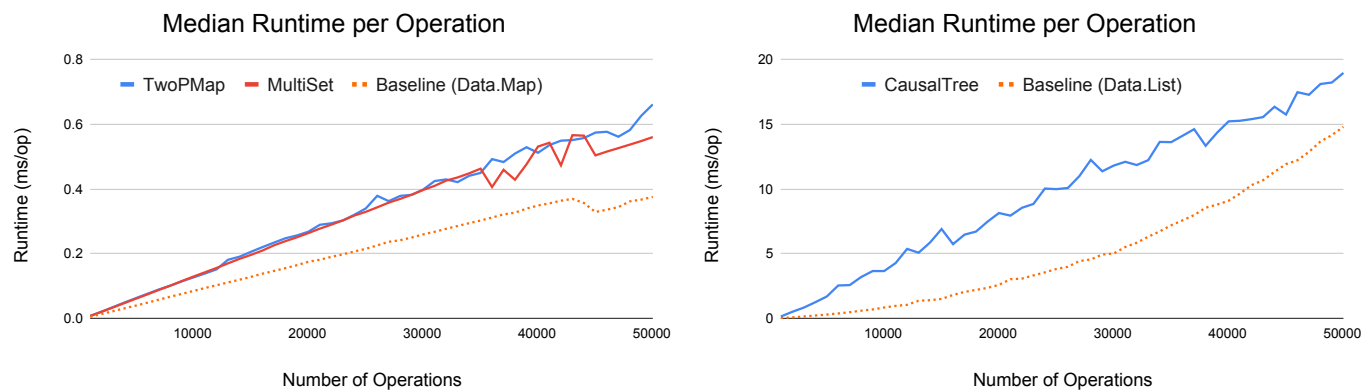

(a) Comparison between the TwoPMap and MultiSet (b) Comparison between the CausalTree VRDT impleVRDT implementations and the Data.Map baseline. mentation and the Data.List baseline.

Fig. 9. Median time per operation vs. the number of operations applied to the data structure, over 11 trials.

machine as described previously in Section 2.3. The slowdown from the Haskell baseline amounts to the cost of the extra bookkeeping operations required by the RDTs, e.g., the maintenance of the tombstone and pending queue in TwoPMap. We also ran experiments on Min, Max, Sum, and LWW, and the per-operation costs were a constant 80ns; no extra bookkeeping is required for these RDTs.

\subsection{Applications}

We built two realistic applications that are backed by VRDT instances: a shared event planner and a collaborative text editor. We close out this section by briefly describing these applications and some of the other infrastructure we built beyond VRDTs to put them together.

Message delivery and UI components. Both of our applications build on Haskell libraries we developed for message delivery and user interfaces. In particular, we developed a message delivery client and server to broadcast un-ordered messages from each client to all other clients. We implemented the server using the servant library with HTTP endpoints for sending messages to broadcast and for listening to a stream of messages from other clients. For the client, we developed an application programming interface (API) which transparently handles network disconnections by buffering and re-sending outgoing messages, abstracting away network interruptions.

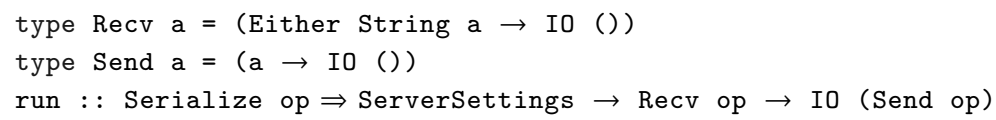

The (simplified) client API entry point is run, which establishes and manages the connection to a server. Applications provide a function that is called whenever a message is received, and run produces a function that applications use to send messages.

Our user interface library is based around functional reactive programming (FRP), a programming paradigm that models values that change over time [Elliott and Hudak 1997]. FRP values are either continuous, called behaviors, or discrete, called events. We treat replicated data types as FRP values whose state changes as a result of actions by the local user or update messages from a remote replica. We use Reflex ${ }^{3}$, a Haskell FRP library, to integrate FRP applications with our message delivery system. Any VRDT instance whose operations can be marshalled and sent over a network, e.g., as JSON, can be used as the state of these distributed applications. We provide the below library function, which internally calls the client API to connect a FRP application client to the server.

${ }^{3}$ https://reflex-frp.org/ 
connectToStore : : (VRDT a, Serialize (Op a), MonadIO m) $\Rightarrow$ ServerSettings $\rightarrow$ a $\rightarrow$ Event (Op a) $\rightarrow$ m (Dynamic a)

connectToStore takes the settings of the server to connect to and an initial state. It also receives an Event of Ops. Any time the FRP client performs an operation, the event fires and this function sends the operation to the server. Dynamic is a special Reflex type that is both an event and a behavior. Whenever its value changes, an event fires as well. Since connectToStore returns a Dynamic of the current state, the FRP application automatically updates its interface whenever an operation is received and applied to the VRDT state.

Event planner. Our shared event planner application allows multiple users to create and manage calendar events and RSVP to event invitations. The planner's state (TwoPMap UniqueId Event) is a two-phase map where elements are the VRDT automatically derived from the Event type described in Figure 7. UniqueId is a pair of ClientId and an integer that is always incremented locally by the client application. It is used to ensure that the keys are unique as required by TwoPMap. The event planner has a terminal interface that supports viewing the list of events, creating events, updating events, and displaying event details. Since the application's state is a VRDT instance, updates are quickly displayed on all clients once they receive the corresponding operations. In this application, 12 lines of code define the types associated with the application's state, and one line of code invokes Template Haskell to generate the operation type for Event and its VRDT instance. The rest of the 400 lines of code in the application implement the user interface. The small amount of code necessary for managing replicated data highlights how VRDTs make it easy to build a distributed application.

Collaborative text editor. Our collaborative text editor represents the state of the text document being edited as a CausalTree. The majority of the code in the text editor (278 lines, out of roughly 350 ) is the causalTreeInput function, which has the following signature:

causalTreeInput : : Dynamic (CausalTree id Char)

$\rightarrow$ Widget (Event (Op (CausalTree id Char)))

causalTreeInput creates a Reflex Widget that builds a text box in the terminal interface that displays the contents of the CausalTree, handles scrolling, and processes keystrokes by the user. It takes a Dynamic of the CausalTree as input so that the view is updated when operations from the network are received. It returns an Event of CausalTree operations that fires whenever keystrokes update the state of the document.

\section{RELATED WORK}

\subsection{Class Specifications in Object Oriented Languages}

Verification-friendly, object-oriented languages, including Spec\# [Barnett et al. 2005], Larch [Guttag et al. 1985], and Dafny [Leino 2010], permit specification and verification of class invariants. Such invariants are encoded as postconditions to class constructors and as pre- and postconditions to methods. Unlike with typeclasses, these languages offer no automated resolution of class instances.

Scala, closer to our work, has typeclass-like features with expressive specifications at the cost of potentially diverging resolution. Using the trait syntax one can define what amounts to a typeclass, as a set of behaviors (akin to Haskell methods) and their type specifications. Using the given syntax, one can define trait instances [Team 2020]. Implicit arguments are used to express Haskell-style type class constraints and thus enable the usage of the trait behaviors. The Scala compiler resolves the implicits to given instances via implicit resolution [Odersky et al. 2017]. Because Scala's type system is so expressive, implicit resolution could be incoherent and diverging. To enforce coherence the compiler uses a disambiguation scheme that relies on a user-provided ranking (§3.5 of Odersky et al. [2017]). Implicit resolution can diverge in theory (§4 of Odersky 
et al. [2017]) but is prevented in practice by employing various heuristics (e.g., breaking infinite loops after five steps), at the cost of potentially brittle results.

\subsection{Verification of Haskell's Typeclass Laws}

Verification of inductive properties, including per-instance typeclass laws, is possible in Haskell using dependently typed features [Eisenberg 2016; Weirich et al. 2019; Xie et al. 2019; McBride 2002]. In work closely related to ours, Scott and Newton [2019] verify algebraic laws of typeclasses using a singletons encoding of dependent types [Eisenberg and Weirich 2012], and they employ generic programming to greatly reduce the proof burden. Even though their generic boilerplate technique is very effective for verifying typeclass instances, it is unclear how the encoding of typeclass laws interacts with the Haskell code that uses those instances. In our approach, typeclass laws are expressed as refinement types and smoothly co-exist with refinement type specifications of clients of typeclass methods. In fact, Scott and Newton initially attempted to use Liquid Haskell, but at the time it was impossible since there was no support for refinement types on typeclasses.

Haskell researchers have developed various techniques outside of Haskell itself to increase their confidence that typeclass laws actually hold. For example, Jeuring et al. [2012] and Claessen and Hughes [2011] used QuickCheck, the property-based random testing tool, to falsify typeclass laws. Zeno [Sonnex et al. 2012] and HERMIT [Farmer et al. 2015] generate typeclass law proofs by term rewriting while HALO [Vytiniotis et al. 2013] uses an axiomatic encoding to verify Haskell contracts. HipSpec [Arvidsson et al. 2016; Claessen et al. 2012] reduces typeclass laws to an external, automated-over-induction theorem prover. hs-to-coq [Spector-Zabusky et al. 2018] converts Haskell typeclasses and instances to equivalent ones in Coq which can then be proved to satisfy the respective laws.

Compared to these approaches, our technique has three main advantages. First, our proofs are Haskell programs, highly automated by SMT and PLE; unlike the other approaches, when proof automation fails, the user does not need to debug the external solver. Second, our proofs co-exist and interact with non-typeclass-specific Haskell code, so Haskell functions can use class laws to prove further properties (as in the assoc2 example of Section 2.2). Finally, our within-Haskell verification approach gives the developer the ability to distinguish between verified and original (i.e., non-verified) typeclasses (as in the Semigroup example of Section 2.2) and the flexibility to only use verified methods on critical code fragments, thus saving verification time.

\subsection{Type System Expressiveness vs. Coherence of Elaboration}

Typeclasses have been adopted by PureScript [Freeman 2017] and have inspired related abstractions in many programming languages, including SML's modules [MacQueen 1984; Dreyer et al. 2007] and Rust's traits [Team Mozilla Research 2017]. These languages (like vanilla Haskell) are not designed for proving rich logical properties, e.g., by making use of dependent types. But such simpler type systems make it possible to implement coherent typeclass resolution; for Haskell in particular, Bottu et al. [2019] prove coherence of GHC's elaboration by showing global uniqueness of dictionary creations. Coherence means that decisions made by typeclass elaboration cannot change the runtime semantics of the program, making it easier to reason about.

Fully dependently-typed languages such as Coq [Sozeau and Oury 2008], Isabelle [Haftmann and Wenzel 2006], Agda [Devriese and Piessens 2011], Lean [de Moura et al. 2015], and F* [Martínez et al. 2019] permit proofs of rich logical properties, and also support typeclasses. However, to maximize expressiveness, their typeclass resolution procedures can end up being divergent or incoherent. For example, in Coq's typeclasses [Sozeau and Oury 2008], instantiation can diverge and is not guaranteed to be coherent since it is not always possible to decide whether two instances overlap [Lampropoulos and Pierce 2018]. 
In our work, we attempt to strike a balance between these two extremes. We use Liquid Haskell's expressiveness to prove typeclass properties, while we use GHC's less expressive type system to perform resolution. This design precludes having distinct typeclasses for two refined types that have the same base type, but this limitation is easily overcome by using newtype to give distinct names to the two refined types, as we saw at the end of Section 2.2. Moreover, our design confers two nice benefits. First, we reuse GHC's mature elaboration implementation. More importantly, using elaboration on the coherent [Bottu et al. 2019], less expressive type system of Haskell, we break the dilemma between expressiveness of the type system and coherence of elaboration.

\subsection{Verifying Replicated Data Types}

No verification can take place without a specification, and precisely specifying the behavior of replicated data types is a significant challenge in itself. Most work proposing new designs and implementations of replicated data structures (e.g., [Shapiro et al. 2011b,a; Roh et al. 2011]) does not provide formal specifications. An exception is Attiya et al.'s work [2016], which precisely specifies a replicated list object and gives a (non-mechanized) correctness proof.

Burckhardt et al. [2014] proposed a comprehensive framework for formally specifying and verifying the correctness of RDTs, using an approach inspired by axiomatic specifications of weak shared-memory models. Although it is not obvious how to automate Burckhardt et al.'s verification approach, the Quelea [Sivaramakrishnan et al. 2015] programming model uses the Burckhardt et al specification framework as a contract language embedded in Haskell that allows programmers to attach axiomatic contracts to RDT operations; an SMT solver analyzes these contracts and determines the weakest consistency level at which an operation can be executed while satisfying its contract. ${ }^{4}$ Gotsman et al. [2016] develop an SMT-automatable proof rule that can establish whether a particular choice of consistency guarantees for operations on RDTs is enough to ensure preservation of a given application-level data integrity invariant. This approach is implemented in Najafzadeh et al.'s CISE tool. Houshmand and Lesani's Hamsaz system [2019] improves on CISE by automatically synthesizing a conflict relation that specifies which operations conflict with each other (whereas this conflict relation has to be provided as input to CISE).

Unlike our approach, tools like Quelea, CISE, and Hamsaz do not, in and of themselves, prove correctness properties of RDT implementations, e.g., strong convergence of replicas. Rather, they determine whether or not it is safe to execute a given RDT operation under the assumption that that replicas satisfy a given consistency policy (in the case of Quelea), or whether or not an applicationlevel invariant will be satisfied, given the consistency policies satisfied by individual operations (in the case of CISE and Hamsaz). The goals of these lines of work are therefore complementary to ours: we prove a property of RDT implementations (strong convergence) that such tools could then leverage as an assumption to prove application-level properties, e.g., that a replicated bank account never has a negative balance. Verification of these application-level properties is important because CRDT correctness alone is not enough to ensure application correctness. (Of course, it would also be possible to prove such application-level properties directly in Liquid Haskell as well.)

Other works [Zeller et al. 2014; Nair et al. 2020; Gomes et al. 2017; Nagar and Jagannathan 2019] directly address proving the correctness of RDT implementations. Zeller et al. [2014] specify and prove SC and SEC for a variety of state-based counter, register, and set CRDTs using the Isabelle/HOL proof assistant. Nair et al. [2020] present an automatic, SMT-based verification tool

\footnotetext{
${ }^{4}$ Implementation-wise, in contrast to our approach which uses Liquid Haskell's solver-aided type system, Quelea is implemented in Haskell by directly querying the underlying SMT solver through the Z3 Haskell bindings at compile time, via Template Haskell.
} 
for specifying state-based CRDTs and verifying application-level properties of them. Neither Zeller et al. nor Nair et al. consider operation-based CRDTs, the focus of this paper.

Gomes et al. [2017] also use Isabelle/HOL to prove SC and SEC; like us, they focus on operationbased CRDTs. In addition to proving that RDT operations commute for three operation-based CRDTs-Shapiro et al.'s counters and observed-remove sets, and Roh et al.'s replicated growable arrays [2011]-Gomes et al. formalize in Isabelle/HOL a network model in which messages may be lost and replicas may crash, and prove that SC and SEC hold (under any behavior of the network model). Although one can extract executable implementations from Isabelle, our semi-automated approach has the advantage that the programmer can write, and use, mechanically verified RDT implementations without ever leaving Haskell. Gomes et al. bake causal delivery of updates into their network model (following Shapiro et al. [2011b], who assume causal delivery of updates in their proof of SEC for operation-baesd CRDTs); however, we observe that causal delivery is neither necessary nor sufficient to guarantee strong convergence [Nagar and Jagannathan 2019].

Nagar and Jagannathan [2019] address the question of automatically verifying strong convergence of various operation-based CRDTs (sets, lists, graphs) under different consistency policies provided by the underlying data store. They develop an SMT-automatable proof rule to show that all pairs of operations either commute or are guaranteed by the consistency policy to be applied in a given order. Given a CRDT specification, their framework will determine which consistency policy is required for that CRDT. Their CRDT specifications are written in an abstract specification language designed to correspond to the first-order logic theories that SMT solvers support, whereas our verified RDTs are running Haskell code, directly usable in real applications.

\section{CONCLUSION}

We have presented an extension of Liquid Haskell to allow refinement types on typeclasses. Clients of a typeclass may assume its methods' refinement predicates hold, while instances of the typeclass are obligated to prove that they do. Implementing this extension was challenged by the fact that Liquid Haskell verifies properties of Core, the intermediate representation of the Glasgow Haskell Compiler, but typeclasses are replaced with dictionaries (records of functions) during translation to Core. Our implementation expands the interaction between Liquid Haskell and GHC to carry over refinements to those dictionaries during verification, and does so in a way that takes advantage of Haskell's typeclass resolution procedure being coherent. We have carried out two case studies to demonstrate the utility of our extension. First, we have used typeclass refinements to encode the algebraic laws for the Semigroup, Monoid, Functor, Applicative, and Monad standard typeclasses, and verified these properties hold of many of their instances. Second, we have used our extension to construct a platform for for distributed applications based on replicated data types. We define a typeclass whose Liquid Haskell type captures the mathematical properties of RDTs needed to prove the property of strong convergence; implement several instances of this typeclass; and use them to build two substantial applications.

\section{ACKNOWLEDGMENTS}

We thank José Calderón for connecting the UCSC and the UMD/IMDEA teams. This material is based upon work supported by the National Science Foundation under Grant Nos. CNS-1563722 and CNS-1801545; by the Defense Advanced Research Projects Agency (DARPA) under Contract No FA8750-16-C-0022; by Comunidad de Madrid BLOQUESCM project No S2018/TCS-4339 and Attractión de Talento No 2019-T2/TIC-13455; and by gifts from Google and Amazon Web Services. Any opinions, findings, conclusions or recommendations expressed in this material are those of the authors and do not necessarily reflect the views of the sponsoring organizations. 


\section{REFERENCES}

Mehdi Ahmed-Nacer, Claudia-Lavinia Ignat, Gérald Oster, Hyun-Gul Roh, and Pascal Urso. 2011. Evaluating CRDTs for Real-Time Document Editing. In Proceedings of the 11th ACM Symposium on Document Engineering (Mountain View, California, USA) (DocEng '11). Association for Computing Machinery, New York, NY, USA, 103-112. https: //doi.org/10.1145/2034691.2034717

Andreas Arvidsson, Moa Johansson, and Robin Touche. 2016. Proving Type Class Laws for Haskell. In International Symposium on Trends in Functional Programming. Springer, 61-74.

Hagit Attiya, Sebastian Burckhardt, Alexey Gotsman, Adam Morrison, Hongseok Yang, and Marek Zawirski. 2016. Specification and Complexity of Collaborative Text Editing. In Proceedings of the 2016 ACM Symposium on Principles of Distributed Computing (Chicago, Illinois, USA) (PODC '16). ACM, New York, NY, USA, 259-268. https: //doi.org/10.1145/2933057.2933090

Mike Barnett, K. Rustan M. Leino, and Wolfram Schulte. 2005. The Spec\# Programming System: An Overview. In Construction and Analysis of Safe, Secure, and Interoperable Smart Devices, Gilles Barthe, Lilian Burdy, Marieke Huisman, Jean-Louis Lanet, and Traian Muntean (Eds.). Springer Berlin Heidelberg, Berlin, Heidelberg, 49-69.

Clark Barrett, Aaron Stump, Cesare Tinelli, et al. 2010. The smt-lib standard: Version 2.0. In Proceedings of the 8th international workshop on satisfiability modulo theories (Edinburgh, England), Vol. 13. 14.

Ken Birman, André Schiper, and Pat Stephenson. 1991. Lightweight Causal and Atomic Group Multicast. ACM Transactions on Computer Systems 9 (08 1991), 272-. https://doi.org/10.1145/128738.128742

Gert-Jan Bottu, Ningning Xie, Koar Marntirosian, and Tom Schrijvers. 2019. Coherence of Type Class Resolution. Proc. ACM Program. Lang. 3, ICFP, Article Article 91 (July 2019), 28 pages.

Sebastian Burckhardt, Alexey Gotsman, Hongseok Yang, and Marek Zawirski. 2014. Replicated Data Types: Specification, Verification, Optimality. In Proceedings of the 41st ACM SIGPLAN-SIGACT Symposium on Principles of Programming Languages (San Diego, California, USA) (POPL '14). Association for Computing Machinery, New York, NY, USA, 271-284. https://doi.org/10.1145/2535838.2535848

Koen Claessen and John Hughes. 2011. QuickCheck: a lightweight tool for random testing of Haskell programs. Acm sigplan notices 46, 4 (2011), 53-64.

Koen Claessen, Moa Johansson, Dan Rosén, and Nicholas Smallbone. 2012. HipSpec: Automating Inductive Proofs of Program Properties.. In ATx/WInG at IFCAR. 16-25.

Robert L Constable and Scott Fraser Smith. 1987. Partial objects in constructive type theory. Technical Report. Cornell University.

Leonardo De Moura and Nikolaj Bjørner. 2008. Z3: An efficient SMT solver. In International conference on Tools and Algorithms for the Construction and Analysis of Systems. Springer, 337-340.

Leonardo de Moura, Soonho Kong, Jeremy Avigad, Floris Van Doorn, and Jakob von Raumer. 2015. The Lean theorem prover (system description). In International Conference on Automated Deduction. Springer, 378-388.

Dominique Devriese and Frank Piessens. 2011. On the bright side of type classes: instance arguments in Agda. ACM SIGPLAN Notices 46, 9 (2011), 143-155.

Derek Dreyer, Robert Harper, Manuel M. T. Chakravarty, and Gabriele Keller. 2007. Modular Type Classes. SIGPLAN Not. 42 , 1 (Jan. 2007), 63-70. https://doi.org/10.1145/1190215.1190229

Richard A. Eisenberg. 2016. Dependent Types in Haskell: Theory and Practice. CoRR abs/1610.07978 (2016). arXiv:1610.07978 http://arxiv.org/abs/1610.07978

Richard A. Eisenberg and Stephanie Weirich. 2012. Dependently Typed Programming with Singletons. SIGPLAN Not. 47, 12 (Sept. 2012), 117-130. https://doi.org/10.1145/2430532.2364522

Conal Elliott and Paul Hudak. 1997. Functional Reactive Animation. In International Conference on Functional Programming. http://conal.net/papers/icfp97/

Andrew Farmer, Neil Sculthorpe, and Andy Gill. 2015. Reasoning with the HERMIT: Tool Support for Equational Reasoning on GHC Core Programs. SIGPLAN Not. 50, 12 (Aug. 2015), 23-34. https://doi.org/10.1145/2887747.2804303

C. J. Fidge. 1988. Timestamps in message-passing systems that preserve the partial ordering. Proceedings of the 11th Australian Computer Science Conference 10, 1 (1988), 56-66. http://sky.scitech.qut.edu.au/ fidgec/Publications/fidge88a.pdf

Phil Freeman. 2017. PureScript by Example. https://doi.org/10.1145/2887747.2804303

GHC 2020. GHC: The Glasgow Haskell compiler. https:/www.haskell.org/ghc/.

Victor B. F. Gomes, Martin Kleppmann, Dominic P. Mulligan, and Alastair R. Beresford. 2017. Verifying Strong Eventual Consistency in Distributed Systems. Proc. ACM Program. Lang. 1, OOPSLA, Article 109 (Oct. 2017), 28 pages. https: //doi.org/10.1145/3133933

Alexey Gotsman, Hongseok Yang, Carla Ferreira, Mahsa Najafzadeh, and Marc Shapiro. 2016. 'Cause I'm Strong Enough: Reasoning about Consistency Choices in Distributed Systems. In Proceedings of the 43rd Annual ACM SIGPLAN-SIGACT Symposium on Principles of Programming Languages (St. Petersburg, FL, USA) (POPL '16). Association for Computing Machinery, New York, NY, USA, 371-384. https://doi.org/10.1145/2837614.2837625 
Victor Grishchenko. 2010. Deep Hypertext with Embedded Revision Control Implemented in Regular Expressions. In Proceedings of the 6th International Symposium on Wikis and Open Collaboration (Gdansk, Poland) (WikiSym '10). Association for Computing Machinery, New York, NY, USA, Article 3, 10 pages. https://doi.org/10.1145/1832772.1832777

John Guttag, James Horning, and Jeannette Wing. 1985. The Larch Family of Specification Languages. Software, IEEE 2 (10 1985), 24-36. https://doi.org/10.1109/MS.1985.231756

Florian Haftmann and Makarius Wenzel. 2006. Constructive type classes in Isabelle. In International Workshop on Types for Proofs and Programs. Springer, 160-174.

Farzin Houshmand and Mohsen Lesani. 2019. Hamsaz: Replication Coordination Analysis and Synthesis. Proc. ACM Program. Lang. 3, POPL, Article 74 (Jan. 2019), 32 pages. https://doi.org/10.1145/3290387

Johan Jeuring, Patrik Jansson, and Cláudio Amaral. 2012. Testing type class laws. In Proceedings of the 2012 Haskell Symposium. 49-60.

Martin Kleppmann and Alastair R Beresford. 2017. A conflict-free replicated JSON datatype. IEEE Transactions on Parallel and Distributed Systems 28, 10 (2017), 2733-2746.

Leslie Lamport. 1978. Time, Clocks, and the Ordering of Events in a Distributed System. Commun. ACM 21, 7 (July 1978), 558-565. https://doi.org/10.1145/359545.359563

Leonidas Lampropoulos and Benjamin C. Pierce. 2018. QuickChick: Property-Based Testing in Coq.

K. Rustan M. Leino. 2010. Dafny: An Automatic Program Verifier for Functional Correctness. In Proceedings of the 16th International Conference on Logic for Programming, Artificial Intelligence, and Reasoning (Dakar, Senegal) (LPAR'10). Springer-Verlag, Berlin, Heidelberg, 348-370.

K Rustan M Leino and Clément Pit-Claudel. 2016. Trigger selection strategies to stabilize program verifiers. In International Conference on Computer Aided Verification. Springer, 361-381.

David MacQueen. 1984. Modules for Standard ML. In Proceedings of the 1984 ACM Symposium on LISP and functional programming. 198-207.

Guido Martínez, Danel Ahman, Victor Dumitrescu, Nick Giannarakis, Chris Hawblitzel, Cătălin Hrițcu, Monal Narasimhamurthy, Zoe Paraskevopoulou, Clément Pit-Claudel, Jonathan Protzenko, et al. 2019. Meta-F*: Proof Automation with SMT, Tactics, and Metaprograms. In European Symposium on Programming. 30-59.

Friedemann Mattern. 1989. Virtual Time and Global States of Distributed Systems. In Proc. Workshop on Parallel and Distributed Algorithms, Cosnard M. et al. (Ed.). North-Holland / Elsevier, 215-226. (Reprinted in: Z. Yang, T.A. Marsland (Eds.), "Global States and Time in Distributed Systems", IEEE, 1994, pp. 123-133.).

Conor McBride. 2002. Faking it Simulating dependent types in Haskell. Journal of functional programming 12, 4-5 (2002), 375-392.

Kartik Nagar and Suresh Jagannathan. 2019. Automated Parameterized Verification of CRDTs. In Computer Aided Verification, Isil Dillig and Serdar Tasiran (Eds.). Springer International Publishing, Cham, 459-477.

Sreeja S. Nair, Gustavo Petri, and Marc Shapiro. 2020. Proving the Safety of Highly-Available Distributed Objects. In Programming Languages and Systems, Peter Müller (Ed.). Springer International Publishing, Cham, 544-571.

Mahsa Najafzadeh, Alexey Gotsman, Hongseok Yang, Carla Ferreira, and Marc Shapiro. 2016. The CISE Tool: Proving Weakly-Consistent Applications Correct. In Proceedings of the 2nd Workshop on the Principles and Practice of Consistency for Distributed Data (London, United Kingdom) (PaPoC '16). Association for Computing Machinery, New York, NY, USA, Article 2, 3 pages. https://doi.org/10.1145/2911151.2911160

Martin Odersky, Olivier Blanvillain, Fengyun Liu, Aggelos Biboudis, Heather Miller, and Sandro Stucki. 2017. Simplicitly: Foundations and Applications of Implicit Function Types. Proc. ACM Program. Lang. 2, POPL, Article Article 42 (Dec. 2017), 29 pages. https://doi.org/10.1145/3158130

Gérald Oster, Pascal Urso, Pascal Molli, and Abdessamad Imine. 2006. Data Consistency for P2P Collaborative Editing. In Proceedings of the 2006 20th Anniversary Conference on Computer Supported Cooperative Work (Banff, Alberta, Canada) (CSCW '06). Association for Computing Machinery, New York, NY, USA, 259-268. https://doi.org/10.1145/1180875.1180916

James Parker, Niki Vazou, and Michael Hicks. 2019. LWeb: Information flow security for multi-tier web applications. Proceedings of the ACM on Programming Languages 3, POPL (2019), 1-30.

Nuno Preguica, Joan Manuel Marques, Marc Shapiro, and Mihai Letia. 2009. A Commutative Replicated Data Type for Cooperative Editing. In Proceedings of the 2009 29th IEEE International Conference on Distributed Computing Systems (ICDCS '09). IEEE Computer Society, USA, 395-403. https://doi.org/10.1109/ICDCS.2009.20

Hyun-Gul Roh, Myeongjae Jeon, Jinsoo Kim, and Joonwon Lee. 2011. Replicated abstract data types: Building blocks for collaborative applications. f. Parallel Distrib. Comput. 71, 3 (2011), 354-368. https://doi.org/10.1016/j.jpdc.2010.12.006

Patrick M. Rondon, Ming Kawaguci, and Ranjit Jhala. 2008. Liquid Types. In Proceedings of the 29th ACM SIGPLAN Conference on Programming Language Design and Implementation (Tucson, AZ, USA) (PLDI'08). Association for Computing Machinery, New York, NY, USA, 159-169. https://doi.org/10.1145/1375581.1375602

John Rushby, Sam Owre, and Natarajan Shankar. 1998. Subtypes for specifications: Predicate subtyping in PVS. IEEE Transactions on Software Engineering 24, 9 (1998), 709-720. 
Ryan G. Scott and Ryan R. Newton. 2019. Generic and Flexible Defaults for Verified, Law-Abiding Type-Class Instances. In Proceedings of the 12th ACM SIGPLAN International Symposium on Haskell (Berlin, Germany) (Haskell 2019). Association for Computing Machinery, New York, NY, USA, 15-29. https://doi.org/10.1145/3331545.3342591

Marc Shapiro, Nuno Preguiça, Carlos Baquero, and Marek Zawirski. 2011a. A comprehensive study of convergent and commutative replicated data types. (2011).

Marc Shapiro, Nuno Preguiça, Carlos Baquero, and Marek Zawirski. 2011b. Conflict-Free Replicated Data Types. In Stabilization, Safety, and Security of Distributed Systems, Xavier Défago, Franck Petit, and Vincent Villain (Eds.). Springer Berlin Heidelberg, Berlin, Heidelberg, 386-400.

Tim Sheard and Simon Peyton Jones. 2002. Template Meta-Programming for Haskell. SIGPLAN Not. 37, 12 (Dec. 2002), 60-75. https://doi.org/10.1145/636517.636528

KC Sivaramakrishnan, Gowtham Kaki, and Suresh Jagannathan. 2015. Declarative Programming over Eventually Consistent Data Stores. In Proceedings of the 36th ACM SIGPLAN Conference on Programming Language Design and Implementation (Portland, OR, USA) (PLDI '15). Association for Computing Machinery, New York, NY, USA, 413-424. https://doi.org/10. $1145 / 2737924.2737981$

William Sonnex, Sophia Drossopoulou, and Susan Eisenbach. 2012. Zeno: An Automated Prover for Properties of Recursive Data Structures. In Tools and Algorithms for the Construction and Analysis of Systems, Cormac Flanagan and Barbara König (Eds.). Springer Berlin Heidelberg, Berlin, Heidelberg, 407-421.

Matthieu Sozeau and Nicolas Oury. 2008. First-Class Type Classes. In Theorem Proving in Higher Order Logics, Otmane Ait Mohamed, César Muñoz, and Sofiène Tahar (Eds.). Springer Berlin Heidelberg, Berlin, Heidelberg, 278-293.

Antal Spector-Zabusky, Joachim Breitner, Christine Rizkallah, and Stephanie Weirich. 2018. Total Haskell is Reasonable Coq. In Proceedings of the 7th ACM SIGPLAN International Conference on Certified Programs and Proofs (Los Angeles, CA, USA) (CPP 2018). Association for Computing Machinery, New York, NY, USA, 14-27. https://doi.org/10.1145/3167092

Bjarne Stroustrup. 1989. Multiple inheritance for C++. Computing Systems 2, 4 (1989), 367-395.

Martin Sulzmann, Manuel MT Chakravarty, Simon Peyton Jones, and Kevin Donnelly. 2007. System F with type equality coercions. In Proceedings of the 2007 ACM SIGPLAN international workshop on Types in languages design and implementation. $53-66$.

Dotty Development Team. 2020. Dotty Documentation. https://dotty.epfl.ch/docs/reference/contextual/type-classes.html

Team Mozilla Research. 2017. The Rust Programming Language. https://www.rust-lang.org/en-US/

Niki Vazou, Joachim Breitner, Rose Kunkel, David Van Horn, and Graham Hutton. 2018. Theorem proving for all: equational reasoning in liquid Haskell (functional pearl). In Proceedings of the 11th ACM SIGPLAN International Symposium on Haskell, Nicolas Wu (Ed.).

Niki Vazou, Eric L Seidel, Ranjit Jhala, Dimitrios Vytiniotis, and Simon Peyton-Jones. 2014. Refinement types for Haskell. In Proceedings of the 19th ACM SIGPLAN international conference on Functional programming. 269-282.

Niki Vazou, Anish Tondwalkar, Vikraman Choudhury, Ryan G. Scott, Ryan R. Newton, Philip Wadler, and Ranjit Jhala. 2017. Refinement Reflection: Complete Verification with SMT. Proc. ACM Program. Lang. 2, POPL, Article Article 53 (Dec. 2017), 31 pages. https://doi.org/10.1145/3158141

Dimitrios Vytiniotis, Simon L. Peyton Jones, Koen Claessen, and Dan Rosén. 2013. HALO: haskell to logic through denotational semantics. In POPL.

Philip Wadler and Stephen Blott. 1989. How to make ad-hoc polymorphism less ad hoc. In Proceedings of the 16th ACM SIGPLAN-SIGACT symposium on Principles of programming languages. 60-76.

Stephanie Weirich, Pritam Choudhury, Antoine Voizard, and Richard A. Eisenberg. 2019. A Role for Dependent Types in Haskell. Proc. ACM Program. Lang. 3, ICFP, Article Article 101 (July 2019), 29 pages. https://doi.org/10.1145/3341705

Stephane Weiss, Pascal Urso, and Pascal Molli. 2009. Logoot: A Scalable Optimistic Replication Algorithm for Collaborative Editing on P2P Networks. In Proceedings of the 2009 29th IEEE International Conference on Distributed Computing Systems (ICDCS '09). IEEE Computer Society, USA, 404-412. https://doi.org/10.1109/ICDCS.2009.75

Hongwei Xi and Frank Pfenning. 1998. Eliminating array bound checking through dependent types. In Proceedings of the ACM SIGPLAN 1998 conference on Programming language design and implementation. 249-257.

Ningning Xie, Richard A. Eisenberg, and Bruno C. d. S. Oliveira. 2019. Kind Inference for Datatypes. Proc. ACM Program. Lang. 4, POPL, Article Article 53 (Dec. 2019), 28 pages. https://doi.org/10.1145/3371121

Peter Zeller, Annette Bieniusa, and Arnd Poetzsch-Heffter. 2014. Formal Specification and Verification of CRDTs. In Formal Techniques for Distributed Objects, Components, and Systems, Erika Ábrahám and Catuscia Palamidessi (Eds.). Springer Berlin Heidelberg, Berlin, Heidelberg, 33-48. 